Article

\title{
Fructose 1,6-bisphosphatase 2-Camk2 interaction is an integral mechanism of long-term potentiation
}

\author{
Przemysław Duda ${ }^{1}$, Tomasz Wójtowicz ${ }^{2}$, Jakub Janczara ${ }^{1,3}$, Daniel Krowarsch ${ }^{4}$, Aleksandra \\ Czyrek $^{4}$, Agnieszka Gizak ${ }^{1}$ and Dariusz Rakus ${ }^{{ }^{*}}$ \\ 1 Department of Molecular Physiology and Neurobiology, University of Wrocław, Wrocław, 50-335, Poland, \\ przemyslaw.duda@uwr.edu.pl (PD), agnieszka.gizak@uwr.edu.pl (AG),dariusz.rakus@uwr.edu.pl (DR) \\ 2 Laboratory of Cell Biophysics, Nencki Institute of Experimental Biology, Polish Academy of Sciences, \\ Warsaw, 02-093, Poland, t.wojtowicz@nencki.edu.pl (TW) \\ 3 Department of Biochemistry and Molecular Biology, Wrocław University of Environmental and Life \\ Sciences, Wrocław, 50-375, Poland, jakub.janczara@upwr.edu.pl (JJ) \\ 4 Department of Protein Biotechnology, University of Wrocław, Wrocław, 50-383, Poland, \\ aleksandra.czyrek@uwr.edu.pl (AC), daniel.krowarsch@uwr.edu.pl (DK) \\ * Correspondence: dariusz.rakus@uwr.edu.pl
}

\begin{abstract}
Long-term potentiation (LTP) is a molecular basis of memory formation. Here, we demonstrate that LTP critically depends on muscle fructose 1,6-bisphosphatase 2 (Fbp2) - a glyconeogenic enzyme and moonlighting protein protecting mitochondria against stress. We show that LTP induction regulates Fbp2 association with neuronal mitochondria and Camk2, and that the Fbp2-Camk2 interaction correlates with Camk2 autophosphorylation. Silencing of Fbp2 expression or simultaneous inhibition and tetramerization of the enzyme with a synthetic effector mimicking the action of physiological inhibitors (NAD ${ }^{+}$and $\mathrm{AMP}$ ) abolishes Camk2 autoactivation and blocks formation of the early phase of LTP and expression of the late phase LTP markers. Astrocyte-derived lactate reduces $\mathrm{NAD}^{+} / \mathrm{NADH}$ ratio in neurons and thus, diminishes the pool of tetrameric and increases the fraction of dimeric Fbp2. We therefore hypothesize that this NAD+-level-dependent increase of the Fbp2 dimer/tetramer ratio might be a crucial mechanism in which astrocyte-neuron lactate shuttle stimulates LTP formation.
\end{abstract}

Keywords: Memory formation, moonlighting protein, protein-protein interaction, astrocyte-neuron lactate shuttle

\section{Introduction}

Long-term potentiation (LTP) in the hippocampus is the most studied example of synaptic plasticity and it is regarded as the neuronal basis of learning [1].

Molecular events underlying the early phase of LTP include glutamate-stimulated $\mathrm{Ca}^{2+}$ influx into a neuron through $\mathrm{N}$-methyl-D-aspartate receptors (NMDAR), subsequent activation of calciumcalmodulin-dependent kinase II (Camk2), and Camk2-dependend insertion of $\alpha$-amino-3-hydroxy5-methylisoxazole-4-propionate receptors (AMPAR) into the post-synaptic membrane [2,3].

The late phase of LTP that encompasses changes lasting from minutes to hours include an induction of expression of a group of immediate early genes such as c-Fos, c-Jun and Arc which participate in different physiological processes associated with synaptic plasticity [4,5].

Although the role of crucial proteins, such as NMDAR, AMPAR and Camk2, and the significance of the astrocyte-derived lactate [6] in the LTP formation has been well established, the precise mechanism underlying the persistent synaptic enhancement is not fully understood.

In this paper, we present a line of evidence that a crucial role in the induction and maintenance of LTP plays fructose 1,6-bisphosphatase 2 (Fbp2). 
$\mathrm{Fbp} 2$ and the second isozyme of fructose 1,6-bisphosphatase, Fbp1, are regulators of gluconeogenesis. However, $\mathrm{Fbp} 2$ plays non-enzymatic functions in a cell due to its interactions with a variety of proteins $[7,8]$. Fbp2 protects mitochondria against the effects of stress stimuli such as elevated calcium levels resulting in mitochondrial swelling and decreased ATP synthesis [7]. Fbp2 also undergoes cytoplasmic-nuclear shuttling in hormone-dependent manners [9], and it has been recently demonstrated that nuclear $\mathrm{Fbp} 2$ regulates mitochondrial biogenesis via interaction with cMyc [10].

Crystallographic studies have revealed that both the Fbp isozymes adopt tetrameric quaternary structure [11]. Nonetheless, it has been shown that in solution, Fbp2 adopts various oligomeric states, and that the dimeric Fbp2 is the main form interacting with mitochondria [12]. It has been demonstrated that the Fbp2 oligomerization is regulated by allosteric effectors (AMP and NAD+) which are indicators of energy and redox states of a cell [13]. Interestingly, it has been shown that oxidation of astrocyte-derived lactate in neurons contribute to memory formation [6] via elevation of $\mathrm{NADH} / \mathrm{NAD}^{+}$ratio [14].

Although neurons are not considered to synthesize glucose or glycogen from non-carbohydrates the expression of both Fbp isozymes has been detected in vertebrate neurons [15]. Intriguingly, the neuronal enzyme is located in dendritic spines and synapses in close vicinity to mitochondria [16]. Unfortunately, because of a lack of isoform-specific antibodies, it is not possible to ultimately conclude which isoform localizes within synaptic terminals.

In the current study, we seek to investigate whether neuronal Fbp function is associated with regulation of mitochondrial polarity and whether it can affect synaptic plasticity in hippocampal neurons.

The results presented here demonstrate for the first time that Fbp2 is an inherent element of machinery of memory formation in the hippocampal structures. We demonstrate that hippocampal neurons express predominantly the Fbp2 isoform and that its colocalization with mitochondria is associated with the LTP induction. Silencing of Fbp2 expression or inhibition/tetramerization of the enzyme decreases mitochondrial polarity and blocks the LTP formation. Moreover, upon the LTP induction Fbp2 interacts with Camk2 and stimulates its autophosphorylation, which is an indispensable step leading to formation of the early phase of the LTP. Intriguingly, the Fbp2 silencing/inhibition also blocks nuclear accumulation of Camk4 and abolishes the expression of LTP late phase markers such as c-Fos and c-Jun.

The capability of Fbp2 to adopt various quaternary conformations in the presence of AMP and $\mathrm{NAD}^{+}$and hence, its ability to interact with different cellular binding partners suggests that Fbp2 may be a decision center linking neurotransmitter signaling with energetic and redox state of brain during formation of memory traces.

\section{Materials and Methods}

\section{Cell culture and LTP induction on cultured cells}

All the procedures were approved by the Representative for the Protection of Animals Used for Scientific or Educational Purposes of the Faculty of Biological Sciences of University of Wroclaw $\left(30^{\text {th }}\right.$ March 2017) and every effort was made to minimize the number of animals used in the experiments.

Hippocampal neurons were isolated from newborn C57BL6 mice and cultured as described previously [17] with a minor modification - the glucose concentration in the culture medium was 2.5 $\mathrm{mM}$. Neurons were seeded at the density of 25,000 cells $/ \mathrm{cm}^{2}$ for in situ hybridization and immunofluorescent experiments, and at the density of 50,000 cells $/ \mathrm{cm}^{2}$ for homogenization or mitochondria isolation. All the experiments were performed using 14-day-old neuronal cultures.

Prior to the LTP induction the cultures were transferred to the Ringer's solution $\left(37^{\circ} \mathrm{C}\right)$ and LTP was induced according to protocol described previously [18,19], with $1 \mu \mathrm{M}$ strychnine and $200 \mu \mathrm{M}$ glycine.

\section{Immunofluorescence}

The immunofluorescence studies were performed as described previously [20]. The cells were incubated overnight at $4^{\circ} \mathrm{C}$ with respective primary antibodies: rabbit anti-Fbp (1:500, isolated and 
purified as described in [21]), mouse anti-Fbp (1:500, Santa Cruz, sc-271799), rabbit anti-Camk2 $\alpha$ (1:500, Sigma Aldrich, C6974), mouse anti-Camk2 $\alpha$ (1:500, ThermoFisher Scientific, MA1-048), mouse antiCamk2 $\alpha$ pT286 (1:300, Abcam, ab171095), rabbit anti-Camk4 (1:500, Sigma Aldrich, HPA017206), mouse anti-Gsk3ß pS9 (1:300, Millipore, 05-643), mouse anti-c-Fos (1:500, ThermoFisher Scientific, MA1-21190), rabbit anti-c-Jun (1:500, ThermoFisher Scientific, 702170), rabbit anti-Map2 (1:500, Sigma Aldrich, M3696), mouse anti-Map2 (1:500, Sigma Aldrich, M4403). The primary antibodies were detected using fluorophore-labelled secondary antibodies: goat anti-mouse-AlexaFluor488 (1:1,000; Abcam, ab150113), goat anti-mouse-AlexaFluor633 (1:1,000; ThermoFisher a21050), goat anti-rabbit-AlexaFluor 488 (1:1,000; Invitrogen, a11034) or goat anti-rabbit-AlexaFluor633 (1:1,000; Invitrogen, a21070). In controls, the primary antibodies were omitted. Nuclei were counterstained with DAPI. To visualize mitochondria the cultures were incubated with $0.1 \mu \mathrm{M}$ MitoTrackerTM Deep Red FM (ThermoFisher Scientific, M22426) or with $2 \mu \mathrm{g} / \mathrm{mL}$ JC-1 Dye (ThermoFisher Scientific, T3168) for $30 \mathrm{~min}$ at $37^{\circ} \mathrm{C}$, prior to the LTP induction.

\section{Fluorescent in situ hybridization (FISH)}

FISH was performed as described previously [20]. The following oligonucleotides complementary to mouse mRNA sequences were used: Fbp1 (5'-[Cyanine5]GACGGGTCCA GCATGAAGCA GTTGACACCA CAATCC-3'), Fbp2 (5'-[Cyanine3]GCACACAGCT GAGATACTCT TGCACATCCT CAGGGGAC-3'). All the oligonucleotides were synthesized by Sigma-Aldrich. In controls, the oligonucleotide probes were omitted.

\section{Inhibitors}

The following inhibitors were used: $5 \mu \mathrm{M}$ or $10 \mu \mathrm{M}$ Fbp inhibitor (5-chloro-2-(N-(2,5dichlorobenzenesulfonamido))-benzoxazole; Cayman Chemicals, 18860), $25 \mu \mathrm{M}$ MCU inhibitor (Ru360; Calbiochem, 557440), $5 \mu \mathrm{M}$ SERCA inhibitor (thapsigargin; Sigma Aldrich, T9033). Each of the inhibitors was added to neuronal culture $30 \mathrm{~min}$ prior to the LTP induction.

\section{Fbp2 expression silencing}

Fbp2 expression was silenced with shRNA against 3'UTR of Fbp2 mRNA (FBP2 MISSIONTM shRNA Lentiviral Transduction Particles; Sigma Aldrich, SHCLNV-NM_007994). The shRNA sequence was: 5'-CCGGAGATGA ATGAGCTATG GAGATCTCGA GATCTCCATA GCTCATTCAT CTTTTTTG-3'. The transduction particles were added to neuronal cultures in the amount of $2.5 \times 10^{5}$ and the transduction was carried out for 72 hours. In controls, the cells were transduced with the nontarget shRNA (MISSIONTM TRC2 pLKO.5 Non-Target shRNA Control Plasmid DNA) at the concentration of $5 \mathrm{ng} / \mathrm{mL}$. The silencing was monitored by immunofluorescent microscopy and WB analysis (Figure 1A-C).

\section{Co-immunoprecipitation}

In the co-immunoprecipitation experiment, recombinant human Fbp2 (purified as described by [12]) and Camk2 $\alpha$ (ThermoFisher Scientific, PR4586C) proteins were used. Briefly, Camk2 $\alpha$ and Fbp2 (final concentrations each of the proteins were $0.37 \mu \mathrm{M}$ ) were mixed and incubated overnight at $4^{\circ} \mathrm{C}$. Next, the mixture was incubated overnight at $4^{\circ} \mathrm{C}$ with $50 \mu \mathrm{g} / \mathrm{mL}$ of antibodies against Fbp or Camk2 $\alpha$ and the complexes were precipitated using $200 \mu \mathrm{L} / \mathrm{mL}$ of the Protein G Agarose (Merck). The precipitates were centrifuged at 4,000 x g for $2 \mathrm{~min}$ and washed with PBS. In control reactions, the precipitating antibodies were omitted. The precipitates were then resuspended in the Laemmli's buffer, resolved by SDS-PAGE, and Western Blot analyses were performed with the use of primary antibodies detecting Fbp when the precipitate was obtained with anti-Camk2 $\alpha$ antibodies, and detecting Camk $2 \alpha$ when the precipitate was obtained using anti-Fbp antibodies.

\section{Preparation of acute brain slices}

Brain slices (350 $\mu \mathrm{m}$ thick) were prepared from C57BL6 mice aged P30-P90, as described in [22]. The slices were cut with McIlwain Tissue Chopper (Ted Pella Inc., USA).

\section{Electrophysiological recordings}

Field recordings (fEPSPs) were performed as described previously [22,23]. All drugs were bathapplied and all recordings were made in CA1 stratum radiatum, in ACSF perfused at $7 \mathrm{~mL} / \mathrm{min}$. Synaptic potentiation was evoked with tetanic stimulation, HFS (4×100 Hz, 1 sec duration, with $10 \mathrm{sec}$ inter-train intervals). Recordings of synaptic currents were performed in cultured primary 
hippocampal neurons, in voltage clamp mode of the patch-clamp technique, as described earlier [24], with modifications. Briefly, holding potential was set at $-60 \mathrm{mV}$, and spontaneous excitatory postsynaptic currents (sEPSCs) were recorded in the Ringer's solution, in the presence of strychnine (1 $\mu \mathrm{M})$ and $5 \mathrm{mM}$ glucose. NMDAR-dependent synaptic potentiation was evoked by bath application of Ringer's solution containing reduced magnesium concentration $(0.5 \mathrm{mM}), 100 \mu \mathrm{M}$ glycine and $30 \mathrm{mM}$ glucose [18]. sEPSCs were recorded in 20 sec sweeps and alteration in sEPSCs frequency, duration and amplitude was expressed as relative change in average sEPSC area per sweep. All control recordings were made in the presence of drug diluents. All electrophysiological data was analyzed in pClamp 10 (Molecular Devices, USA) software package.

\section{Biolayer interferometry}

Measurements of the kinetics of Fbp2-Camk2a interaction was performed using ForteBio Octet K2 (Pall ForteBio, Fremont, CA) and high-specificity anti-His antibody biosensor (His2, Pall ForteBio, Fremont, CA). Studies were performed at $25^{\circ} \mathrm{C}$ with shaking at 1,000 rpm in PBS supplemented with 2 $\mathrm{mM} \mathrm{Mg}^{2+}$ and $10 \mu \mathrm{M} \mathrm{Ca}^{2+}$. Sensor tips were hydrated in buffer for $30 \mathrm{~min}$ prior to use. The 96-microwell plates were filled with $200 \mu \mathrm{L}$ of buffer or samples and incubated for $10 \mathrm{~min}$ prior measurements for system stabilization. Camk $2 \alpha(3.5 \mu \mathrm{g} / \mathrm{ml})$ was loaded on the His2 sensor for $120 \mathrm{sec}$ and washed for 60 sec. A reference sensor without Camk2 $\alpha$ served as a background control. Association and dissociation phases (300 sec each) were monitored at various concentrations of $\mathrm{Fbp} 2$ protein ranging from 50 to 400 nM. Kinetic parameters were determined by global fitting with the 1:1 model. Response values from the last $10 \mathrm{sec}$ of the association phase were averaged and used for equilibrium dissociation constants calculation. Data were analyzed with ForteBio Data Analysis 11.0 software (Pall ForteBio, San Jose, CA, USA).

\section{Thermophoresis}

Fbp2-Camk2 $\alpha$ interaction was studied using microscale thermophoresis with the NanoTemper Monolith NT.115 instrument (NanoTemper Technologies GmbH). Camk2a was labeled with the Monolith His-Tag Labeling Kit RED tris-NTA 2nd Generation (Nanotemper Technologies) according to the manufacturer's instruction. Various concentrations of Fbp2 $(0.397 \mathrm{nM}-13 \mu \mathrm{M})$ were titrated against labeled Camk2 $\alpha$ ( $50 \mathrm{nM}$ ) in PBS buffer, supplemented with $0.05 \%$ Tween. Samples were loaded into the Premium Coated Capillaries (NanoTemper Technologies $\mathrm{GmbH}$ ) and thermophoresis was measured using $80 \%$ LED power and $40 \%$ infrared laser power in the ambient temperature of $23^{\circ} \mathrm{C}$. F0 and $\mathrm{F} 1$ times were $-1-0 \mathrm{sec}$ and $1.1-2.1 \mathrm{sec}$, respectively. Datasets were processed with the MO.Affinity Analysis software (NanoTemper Technologies GmbH).

\section{In situ detection of protein interaction}

Endogenous detection of Fbp-Camk2 $\alpha$ interaction was performed using the Duolink® In Situ Orange Starter Kit Mouse/Rabbit (Sigma-Aldrich) according to the protocol provided by the manufacturer, using mouse anti-Fbp (1:500, Santa Cruz, sc-271799) and rabbit anti-Camk2 $\alpha$ (1:500, Sigma Aldrich, C6974) primary antibodies. In controls, the primary antibodies were omitted.

\section{Western blot}

Western blot of neuronal protein extracts was performed as described in [20] using the following antibodies: rabbit anti-Fbp (1:2,000, [21]), mouse anti-Camk2 $\alpha$ (1:1,000, ThermoFisher Scientific, MA1048), and rabbit anti-tubulin $\beta 3(1: 1,000$, Synaptic System, 302302). Goat anti-rabbit peroxidaseconjugated (1:50,000, Merck, a0545) and goat anti-mouse peroxidase-conjugated antibodies (1:50,000, Merck, a9044) were used as secondary antibodies and the reaction was visualized using the SuperSignal ${ }^{\mathrm{TM}}$ West Pico PLUS Chemiluminescent Substrate (ThermoFisher).

\section{Isolation of mitochondria and measurement of mitochondrial swelling and polarization}

The neuronal mitochondria were purified on sucrose gradient and swelling of the organelles was monitored as an abrupt decrease in light absorbance at $540 \mathrm{~nm}$ as described in [7]. To determine the polarization of the organelles the JC-1 Dye (ThermoFisher Scientific, T3168) was used. The experiment was performed as described in [12]. The data was expressed as a ratio of JC-1 fluorescence intensities at 590 and $530 \mathrm{~nm}$.

\section{Confocal microscopy and fluorescence analysis}


Confocal microscopy analysis was performed as described previously [20]. The quantification of the fluorescence was carried out using the ImageJ software [25]. For the analysis of protein and mRNA expression the mean fluorescence intensity was measured from regions of interest (ROIs). The ROIs were defined based on the Map2 immunostaining which delineates the neuronal cells. The mean Camk4 fluorescence intensity was measured in the areas of ROIs, or in the nuclei area of Map2-positive cells, and was normalized as a ratio relative to the mean intensity of Map2 staining in the same ROIs [26]. In the immunodetection and in situ hybridization experiments, $\mathrm{N}$ stands for number of ROIs analyzed.

For the analysis of Fbp-mitochondria colocalization the Manders' coefficient was used. The coefficient varies from 0 (no co-localization) to 1 (100\% of co-localization). It was determined as the ratio of summed intensities of pixels from the Fbp image for which the intensity in the mitochondrial channel was above zero to the total intensity in the Fbp channel [27].

\section{Statistical analysis}

Data was analyzed using Microsoft Excel 2016. Results are expressed as a mean and standard deviation. If not stated otherwise, data was checked for normality using the Shapiro-Wilk test, and for the evaluation of statistical significance the two-tailed Student's T-test was used. A probability of $p$ $<0.05$ was considered to represent a significant difference. All the experiments were done at least in triplicate. 


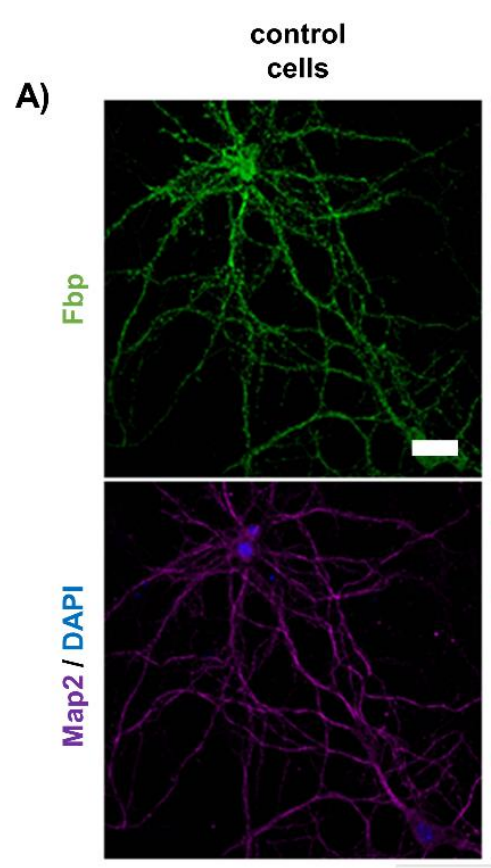

B)

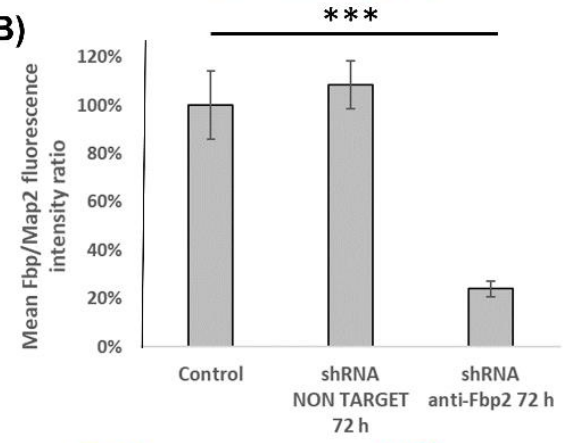

D)
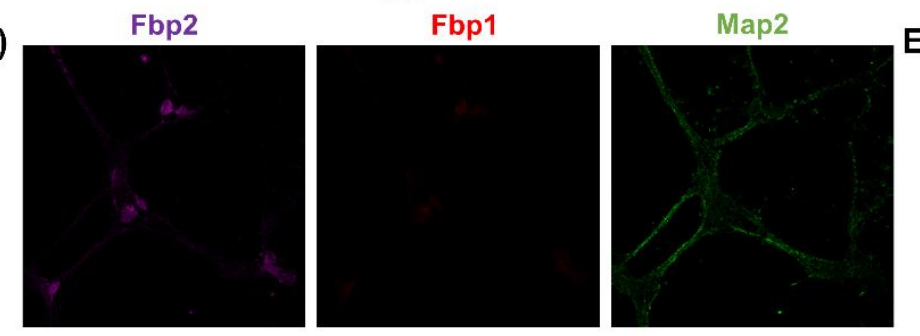

F)

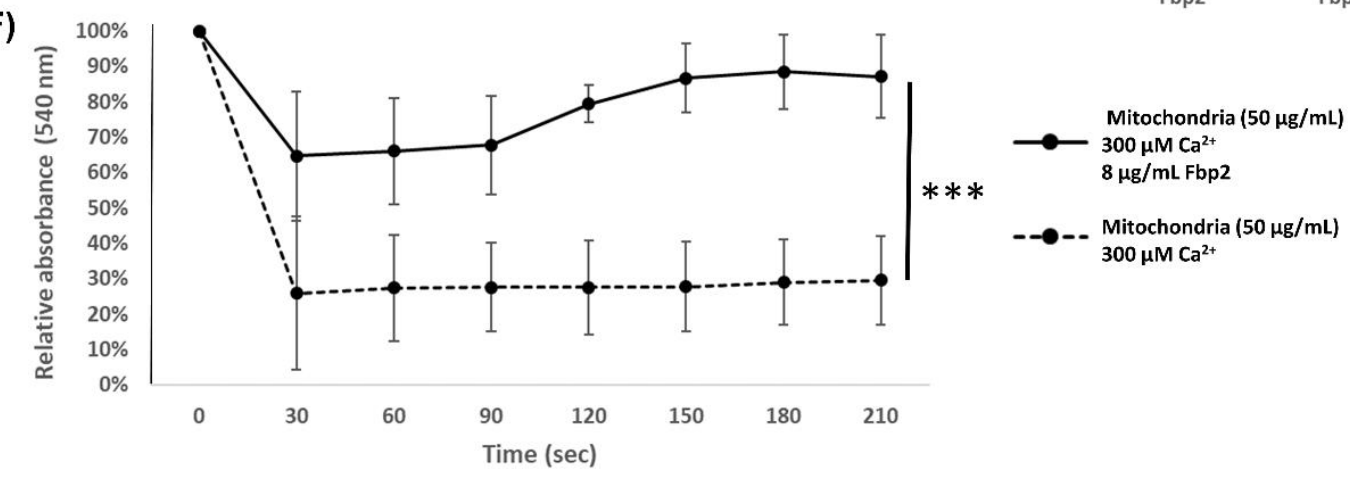

C)

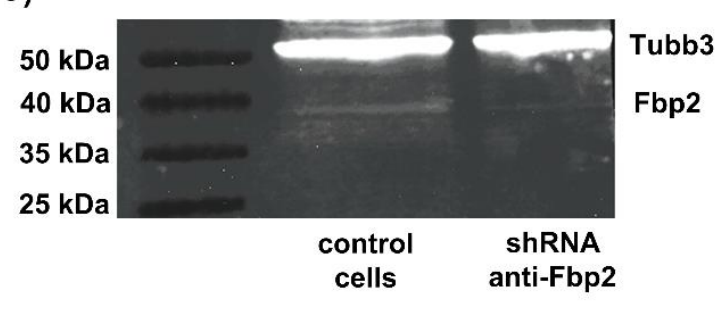

E)

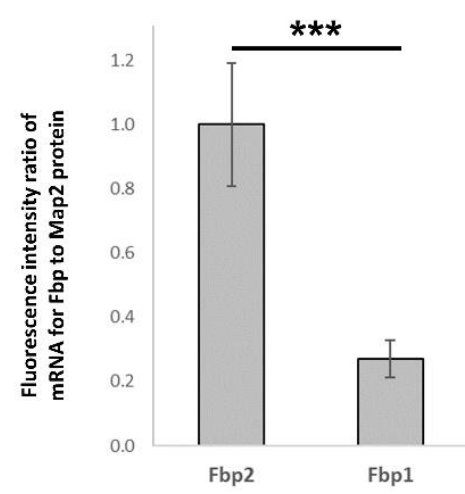

Figure 1. A), B) and C) Silencing of Fbp2 expression in cultured hippocampal neurons. A) Expression of Fbp in hippocampal neurons: untreated (left image), treated with antisense shRNA (middle image) and shRNA against Fbp2 (right image). Bar=20 $\mu$, $\mathrm{N}=30$ ROIs. B) Decrease in Fbp2-associated fluorescence (expressed as the ratio of Fbp2 to Map2 fluorescence). C) Western Blot analysis of Fbp2-silenced hippocampal neurons. D) and E) Fbp2 is the main isoform of Fbp in hippocampal neurons. D) In situ hybridization demonstrates that fluorescent signal associated with mRNA for Fbp2 is much stronger than for Fbp1. E) 
Quantification of the mRNA for Fbp2 and Fbp1. Bar=20 $\mu$, N=30 ROIs. F) Fbp2 protects neuronal mitochondria against calciuminduced swelling. The amount of swollen mitochondria is inversely proportional to the decrease of relative absorbance at $540 \mathrm{~nm}$. The absorbance measured before $\mathrm{Ca}^{2+}$ addition was assumed to be $100 \%$.

The values are given as a mean and SD. ${ }^{* * *}$ indicates $\mathrm{p}<0.001$.

\section{Results}

\section{Fbp2 colocalization with neuronal mitochondria}

To explore the potential involvement of $\mathrm{Fbp}$ in regulation of neuronal mitochondria polarization and synaptic plasticity we used cultured mouse hippocampal neurons and immunofluorescent techniques. In control conditions, about half of the Fbp-associated fluorescent signal colocalized with mitochondrial staining (Figure 2A,B). Because neurons may express both Fbp isoforms [15] we assessed the ratio of $\mathrm{Fbp} 2$ to $\mathrm{Fbp} 1$ using in situ hybridization technique which revealed that Fbp2 accounted for approximately $75 \%$ of the total neuronal Fbp (Figure 1D,E). Since our previous study has demonstrated that $\mathrm{Fbp} 2$, but not $\mathrm{Fbp} 1$, interacts with and protects cardiomyocytic mitochondria against swelling [28] and since Fbp2 silencing did not influence expression of mRNA for Fbp1 (data not shown), thus, it can be assumed that practically all changes in the localization of Fbp shown in this study, were related to Fbp2.

Chemical induction of LTP stimulated time-dependent changes in the colocalization of the Fbp2and mitochondria-related signals (Figure 2B,C). During the first $4 \mathrm{sec}$ after beginning of the LTP induction we observed a significant decrease of the $\mathrm{Fbp}$ signal associated with mitochondria (Figure 2B,C) but in the next $4 \mathrm{sec}$, the Fbp-mitochondria colocalization increased by approx. $60 \%$ and persisted for the next 60 minutes (Figure 2B,C).

LTP is initiated by $\mathrm{Ca}^{2+}$ influx through NMDARs [29]. It has been demonstrated that calcium ions must first enter mitochondria in order to activate Camk2 in a ROS- and $\mathrm{Ca}^{2+}$ - dependent manner [30,31]. On the other hand, a prolonged modestly elevated level of cytosolic $\mathrm{Ca}^{2+}$ inhibits LTP formation [32]. Since it has been demonstrated that $\mathrm{Fbp} 2$ binds to cardiomyocyte mitochondria under Gsk3 $\beta$ inhibition or elevation of cellular $\mathrm{Ca}^{2+}$ levels [7] we tested whether and which calcium stores can regulate interaction of neuronal $\mathrm{Fbp} 2$ with mitochondria.

As it could be expected, the presence of calcium in the extracellular medium was prerequisite to observe the LTP-related changes in Fbp2-mitochondria colocalization (Figure 2F) and absence of $\mathrm{Ca}^{2+}$ in the extracellular solution, or chelation of the cation with EGTA (Figure 2F) resulted in much weaker interaction of the enzyme with mitochondria.

In the presence of $\mathrm{Ca}^{2+}$ in the external solution, the inhibition of mitochondrial calcium uptake with Ru360 [33] was also associated with a very low level of the Fbp-mitochondria colocalization and induction of LTP in such conditions had no effect on the subcellular distribution of the enzyme (Figure 2E). On the other hand, inhibition of sarco/endoplasmic reticulum $\mathrm{Ca}^{2+}$-ATPase (SERCA) with thapsigargin, which is known to elevate concentration of cytosolic $\mathrm{Ca}^{2+}$ [34], strongly stimulated the Fbp2 binding to mitochondria (Figure 2E). Since it is well established that the lack of calcium in the extracellular fluid or application of Ru360 or thapsigargin inhibits LTP formation [35-37] thus, our results suggest that mitochondrial $\mathrm{Ca}^{2+}$ uptake correlates with the Fbp2 binding to the organelles, however, such an association is not sufficient for the LTP formation. 

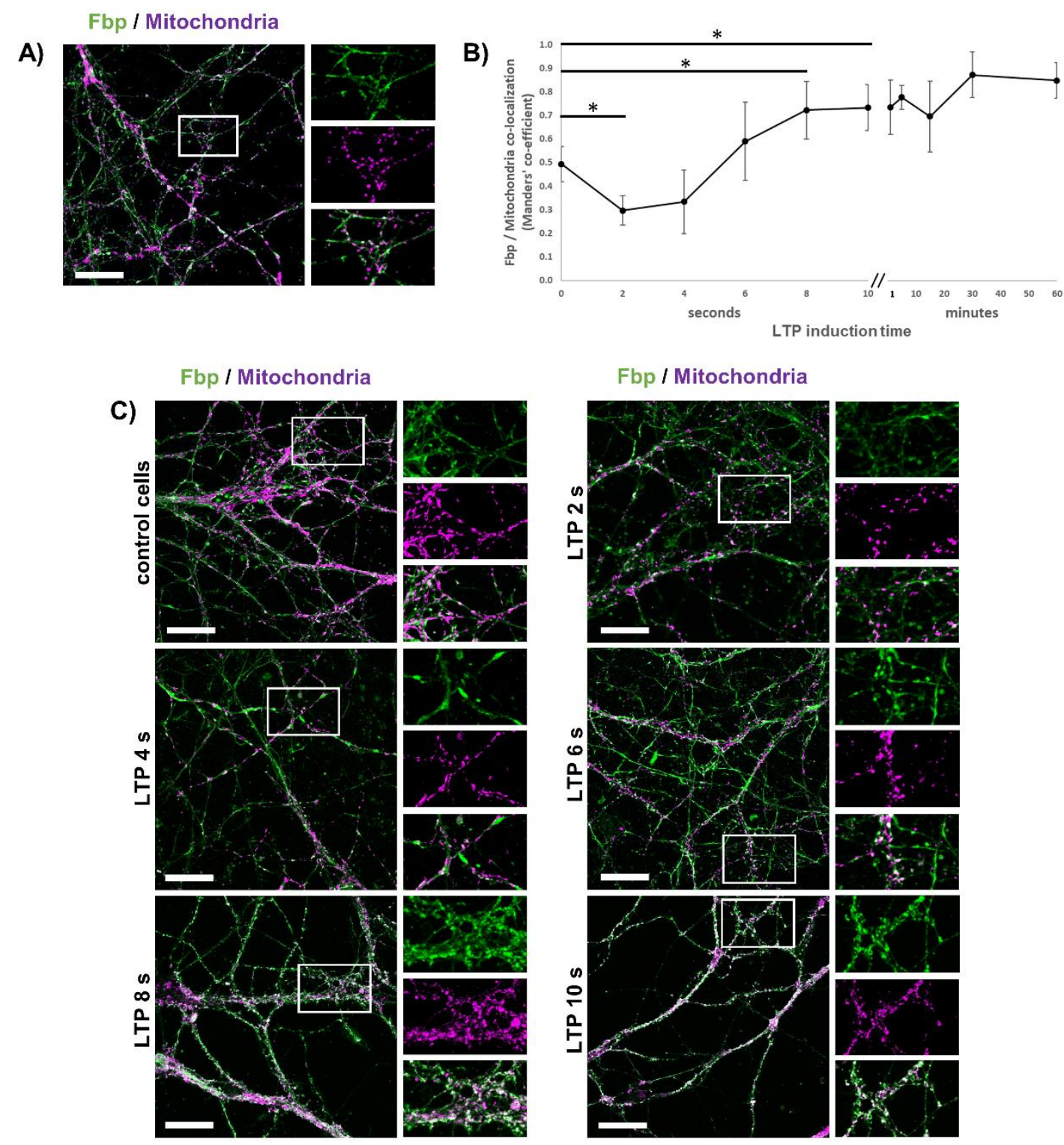

D)

E)

F)
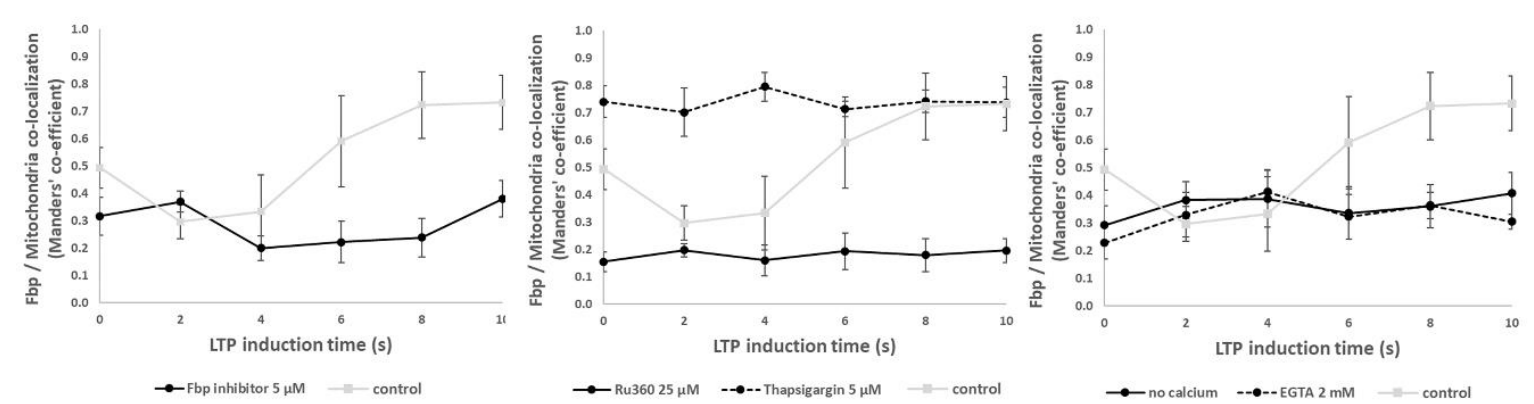

-----EGTA $2 \mathrm{mM}-$ - control

Figure 2. Fbp colocalizes with neuronal mitochondria in a calcium-dependent manner.

A) and B) In control (unstimulated) neurons, about half of Fbp molecules colocalize with mitochondria. B) and C) Stimulation of LTP decreases the colocalization in the first 4 sec but subsequently, the colocalization gradually increases again. D) Fbp inhibition/tetramerization decreases colocalization of the enzyme with mitochondria both in unstimulated and in stimulated neurons. E) Inhibition (with Ru360) of $\mathrm{Ca}^{2+}$ uptake by mitochondria results in decreased Fbp-mitochondria colocalization while 
block (using thapsigargin) of $\mathrm{Ca}^{2+}$ uptake to endoplasmic reticulum results in increase of the colocalization. F) Absence of $\mathrm{Ca}^{2+}$ in the extracellular solution or chelation of the cations decreases Fbp-mitochondria co-localization both in unstimulated and in stimulated neurons.

Bar $=10 \mu \mathrm{m}$. The values are given as a mean and SD. * indicates $\mathrm{p}<0.05$. For each time point the colocalization was measured from 40 images.

Basically, induction of LTP results in a long-lasting mitochondria depolarization [38] which is associated with a phasic occurrence of mitoflashes, transient events of higher mitochondrial activity comprising mitochondrial depolarization and reactive oxygen species production [39]. Results presented here demonstrated, however, that mitochondria underwent hyperpolarization during the first $4 \mathrm{sec}$ after beginning of the LTP induction (Figure 3A,B). Such a short $(<4 \mathrm{sec})$ and transient hyperpolarization of mitochondria upon the LTP induction/glutamate stimulation has never been observed earlier. It presumably reflects a transient elevation of cytosolic $\mathrm{Ca}^{2+}$ concentration after influx of the ions via NMDAR but before their buffering by mitochondria. It has been previously shown that in neurons treated with $0.1 \mathrm{mM}$ NMDA, the increases in cytosolic and in mitochondrial $\mathrm{Ca}^{2+}$ were virtually identical [40]. However, we did not use any exogenous NMDAR agonist to induce LTP $[18,19]$ so the opening of NMDARs was mediated only by endogenous glutamate which titer was presumably many times lower than the concentration of NMDA used in the previous study. Thus, this is likely that in our experimental model, the rate of $\mathrm{Ca}^{2+}$ influx into cytosol via NMDAR was lower and there was a lag in the mitochondrial cation uptake which in turn, induced a transient mitochondrial membrane hyperpolarization event.

Importantly, we observed that during the hyperpolarization event (Figure 3A,B), the colocalization of $\mathrm{Fbp} 2$ with mitochondria was significantly decreased (Figure 2B). $4 \mathrm{sec}$ after beginning of the LTP induction we could see a fast decrease in the polarization (Figure $\mathbf{3 A}, \mathbf{B}$ ) and it was synchronized in time with an increased association of $\mathrm{Fbp} 2$ with mitochondria (Figure 2B).

A reciprocal correlation between the Fbp2-mitochondria colocalization and mitochondrial membrane potential was also observed when neurons were treated with Ru360 (Figure 2E and Figure 3A). On the other hand, in the presence of thapsigargin, the association of Fbp2 with the organelles was significantly elevated but the mitochondrial polarity was unaffected (Figure 2E and Figure 3A).

In the presence of both inhibitors, the induction of LTP did not change the basal Fbp2 association with mitochondria and the mitochondrial polarization was only moderately decreased (Figure 2E and Figure 3A).

Our previous experiments have demonstrated that $\mathrm{Fbp} 2$ not only associates with mitochondria in the $\mathrm{Ca}^{2+}$-dependent manner but it also protects them against calcium-induced swelling [7] and basically, we observed the same effect studying mitochondria from the hippocampal neurons culture (Figure 1F). Since we have also shown that dimeric Fbp2 is the preferred oligomeric form of the enzyme for interactions with mitochondria [12], we asked whether and which oligomeric state of Fbp2 is associated with the changes in the polarization observed during the LTP induction. Because binding of allosteric inhibitors, such as AMP and $\mathrm{NAD}^{+}$, to $\mathrm{Fbp}$ induces and/or stabilizes the tetrameric conformation of the enzyme [11,41] we used a synthetic Fbp inhibitor, 5-chloro-2-(N-(2,5dichlorobenzenesulfonamido))-benzoxazole (further called iFbp), which mimics the action of the physiological allosteric inhibitors [42,43].

After incubation of neurons with iFbp we observed a low level of the Fbp2-mitochondria colocalization which correlated with a strong depolarization of mitochondrial membrane. Both phenomena were not affected by the LTP induction (Figure 2D and Figure 3A). Similarly strong depolarization of mitochondria we observed after partial silencing of Fbp2 expression with siRNA (Figure 3A). This reinforces the hypothesis that the Fbp2 interaction with mitochondria is essential for the proper polarization of the organelles in neurons, both during resting state and after LTP induction. 
A)

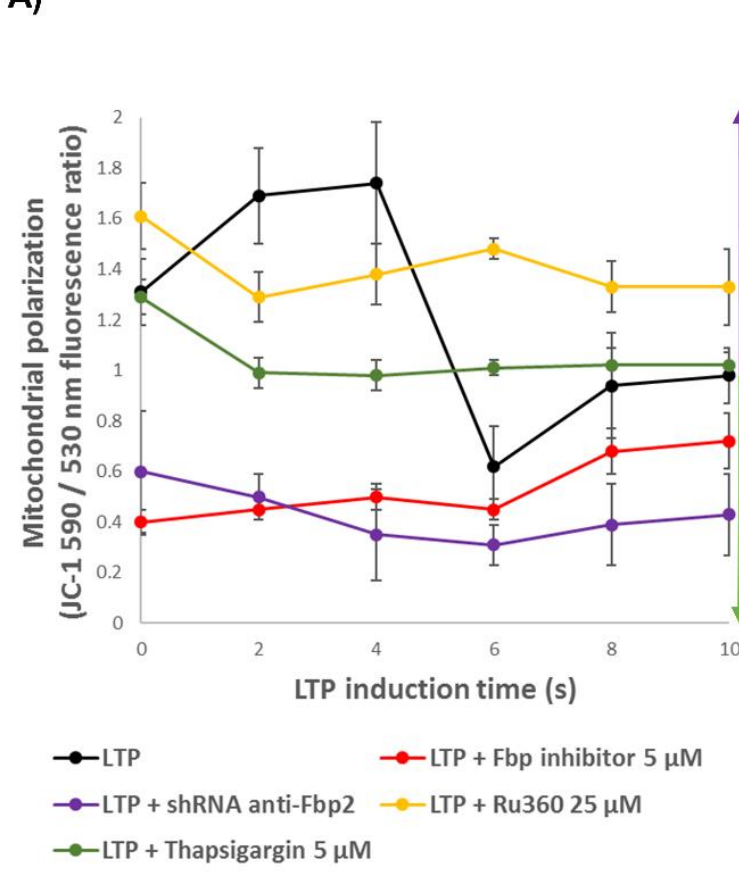

B)

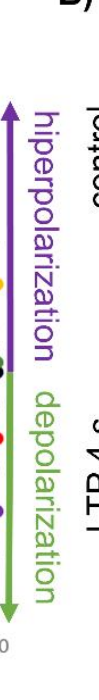

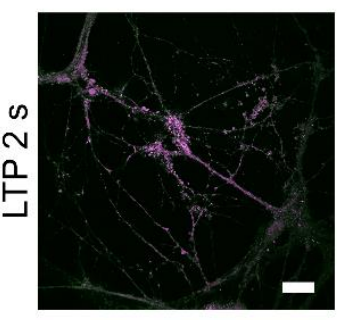
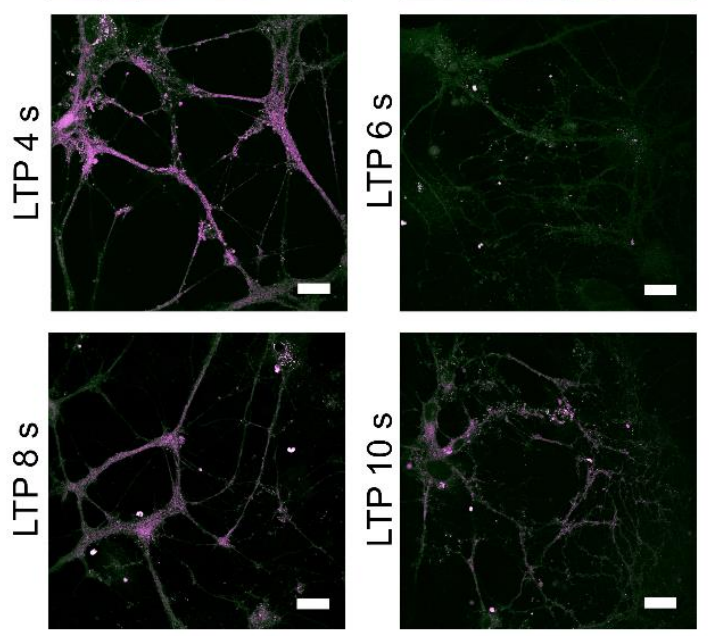

Figure 3. LTP-associated changes in mitochondrial membrane polarization.

A) Induction of LTP results in a short event of mitochondrial hyperpolarization followed by a period of their depolarization. Inhibition/tetramerization of $\mathrm{Fbp}$ (red line) as well as its silencing (blue line) results in depolarization of mitochondria. In the presence of thapsigargin (an inhibitor of the sarco/endoplasmic reticulum $\mathrm{Ca}^{2+}$-ATPase; green line) or Ru360 (an inhibitor of $\mathrm{Ca}^{2+}$ uptake to mitochondria; yellow line), mitochondria are weakly hyperpolarized and depolarized, respectively, as compared to basal polarization (at time 0 ).

Except the control conditions (no inhibition/tetramerization or silencing of Fbp; black line), the induction of LTP does not affect mitochondrial polarization. B) Time-dependent changes in mitochondrial polarization after the LTP induction. Bar $=20 \mu \mathrm{m}$. The values are given as a mean and SD. N=30 ROIs.

\section{The effect of Fbp2 on LTP formation}

Fbp2 silencing or inhibition/tetramerization significantly decreased neuronal mitochondria polarization and made it insensitive to LTP induction. Thus, we checked whether the manipulation with $\mathrm{Fbp} 2$ quaternary structure (using iFbp) or concentration (siRNA-mediated silencing) may affect functional aspects of synaptic potentiation in excitatory synapses. Firstly, we analyzed spontaneous excitatory postsynaptic currents (sEPSCs) in cultured hippocampal neurons. We found that in control conditions, pharmacological induction of NMDAR-dependent synaptic potentiation resulted in a robust upregulation of sEPSC amplitude and frequency, and enlarged an average sEPSC area (Figure 4A,B). When iFbp $(5 \mu \mathrm{M})$ was applied, the basal sEPSC area was not affected (Figure $4 \mathbf{B})$, however, LTP was significantly impaired.

Next, we silenced Fbp2 expression in hippocampal neurons. As shown in Figure 4C, in control neurons transduced with non-target sequence shRNA, the LTP induction resulted in an upregulation of sEPSCs. However, when Fbp2 expression was silenced, LTP was abolished (Figure 4C).

To determine if Fbp2 is required for LTP in hippocampal network, we recorded field excitatory postsynaptic potentials (fEPSPs) in the CA1 region, in response to Shaeffer collateral electrical stimulation, in the absence and presence of iFbp. We found that in control conditions, high-frequency electrical stimulation resulted in robust and stable LTP in excitatory synapses, over a period of 90 min (Figure 4D,E). The application of iFbp had no impact on basal fEPSPs (Figure 4E). However, the electrically induced LTP was completely abolished in the presence of iFbp (Figure 4D,E). Altogether, this demonstrates that the presence of Fbp2 is critical to support NMDAR-dependent functional scaling of excitatory synapses both in vitro and ex vivo. 
A) B)
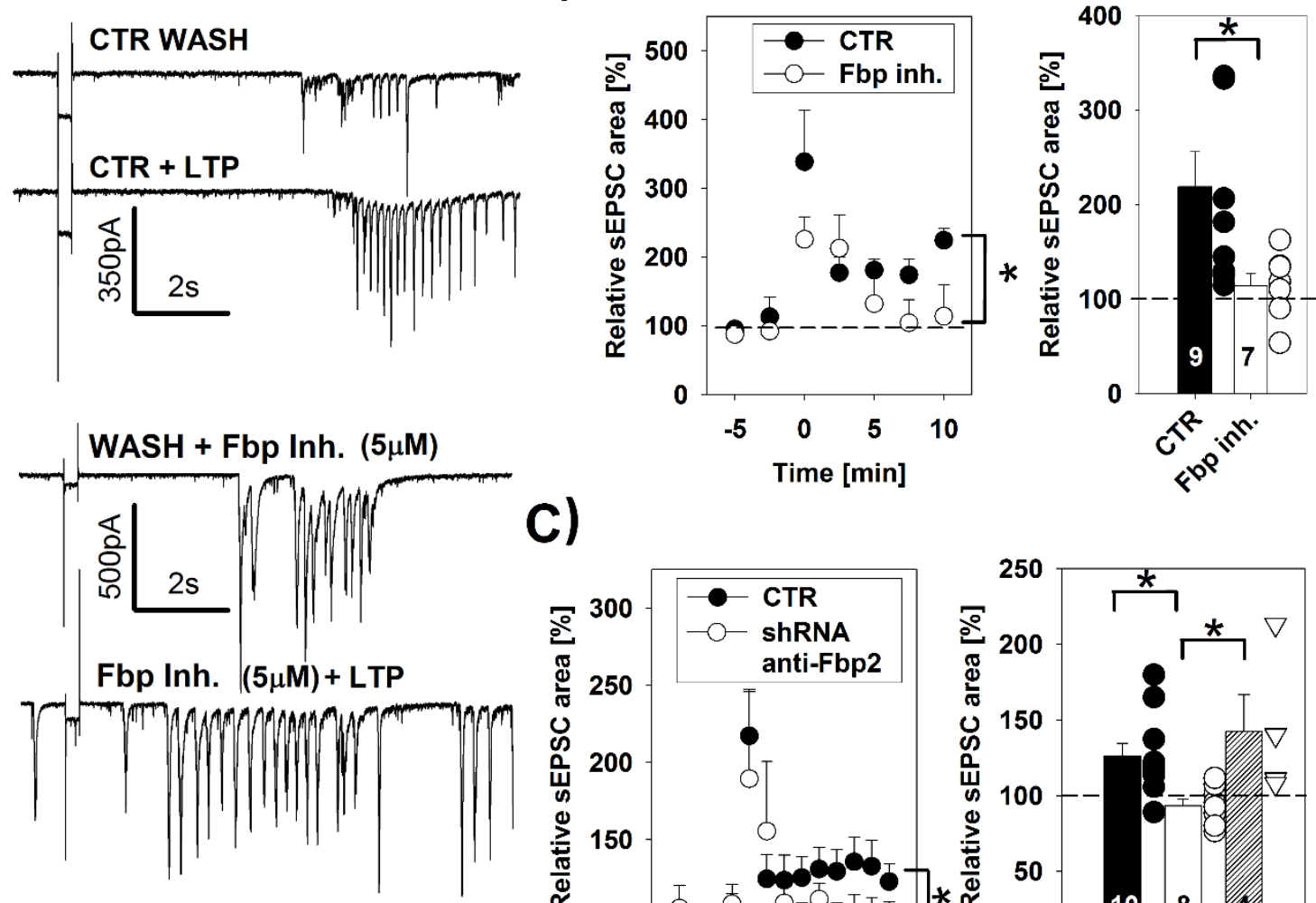

c)
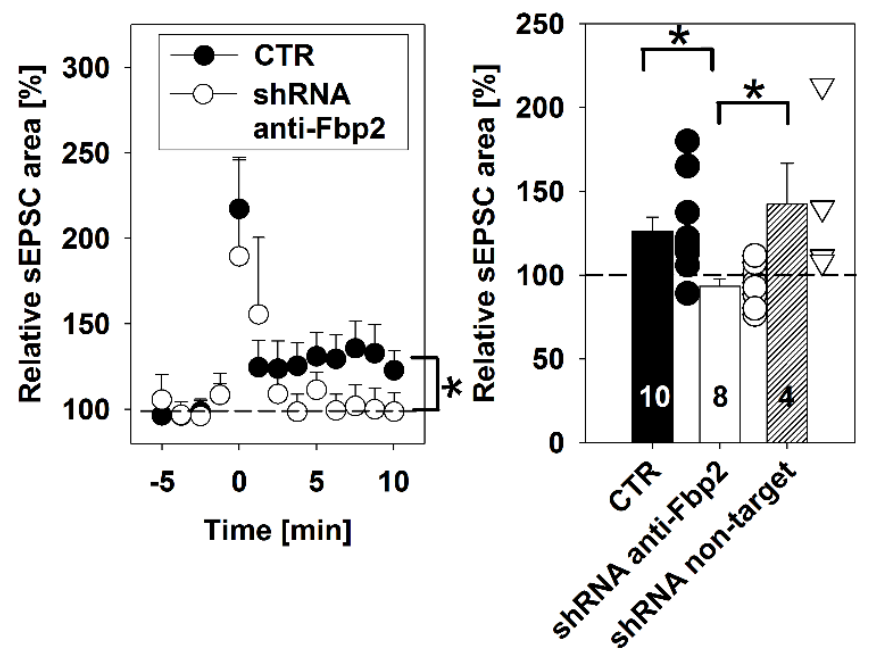

D)

E)
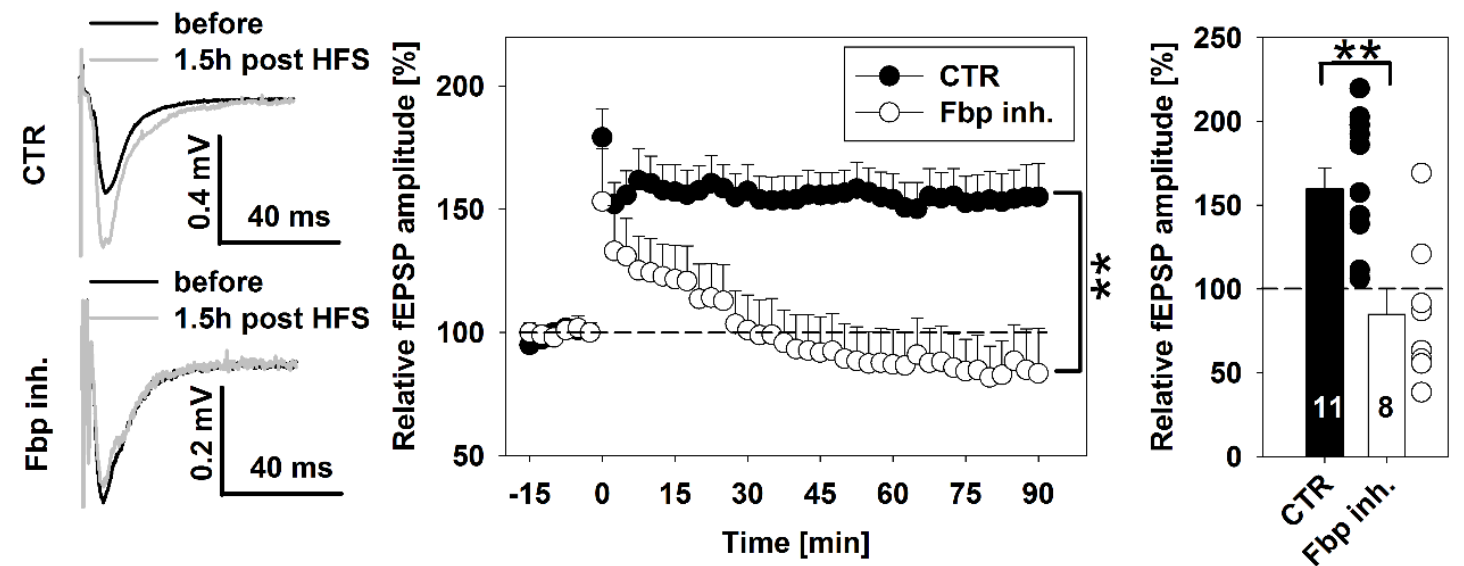

Figure 4. Impact of Fbp2 on synaptic potentiation of excitatory synapses.

A) Exemplary traces of sEPSCs in hippocampal cultures. sEPSCs were recorded continuously before ("WASH") and for 10 min following pharmacological induction of LTP. The upregulation of frequency and amplitude of sEPSCs is more pronounced in control conditions (upper panel), as compared to that observed in the presence of Fbp inhibitor (lower panel). B) Time course (left panel) and relative sEPSC area change $10 \mathrm{~min}$ post LTP induction (right panel) in control neurons (black), and neurons incubated with the Fbp inhibitor (white). In the presence of the inhibitor, potentiation of sEPSC is significantly reduced (unpaired Student's t-test, $\mathrm{N}=3$ cultures, $\mathrm{p}<0.05$; numbers on bars indicate individual neurons recorded). C) Time course (left panel) and relative sEPSC area change $10 \mathrm{~min}$ post LTP induction (right panel) in control neurons (black), and neurons transduced with shRNA against Fbp2 (white) or non-target sequence (dashed white). The silencing of Fbp2 abolishes changes in sEPSC relative area following LTP as compared to control group and shRNA non-target group (One-Way ANOVA with Holm-Sidak post-hoc, N=3 cultures, F2,21=4,964, $\mathrm{p}<0.05$; numbers on bars indicate individual neurons recorded). D) 
Exemplary traces of fEPSPs evoked in mouse acute hippocampal slices, at CA1 stratum radiatum in response to stimulation of Schaffer collaterals before (black traces) and $1.5 \mathrm{~h}$ post high frequency stimulation (HFS). Note that in contrast to control slices, presence of the Fbp inhibitor $(10 \mu \mathrm{M})$ abolishes changes in fEPSPs post HFS (grey traces). E) The time course (left panel) and average fEPSP amplitudes recorded 90 min post HFS in control slices (black), and following incubation with the Fbp inhibitor throughout recordings (white). Note that the inhibitor significantly abolishes LTP magnitude (unpaired Student's t-test, N=5 mice per group, $\mathrm{p}<0.01$; numbers on bars indicate individual slices recorded). Asterisk indicate significant difference. Error bars indicate SEM.

Afterwards, we verified the electrophysiological results by monitoring changes in fluorescence signals associated with protein markers of both LTP phases.

To monitor the induction of the early phase, we checked phosphorylation status of proteins crucial for the formation of LTP such as Camk2 and AMPA receptor (AMPAR). Autophosphorylation leading to autoactivation of Camk2 (Camk2 $\alpha$ pT286) is considered as one of the first events in the canonical pathway of memory formation [44]. Phosphorylation of AMPAR subunits by Camk2 stimulates excitatory synaptic plasticity by incorporation of the receptor into the postsynaptic membrane [45]. We also searched for the presence of the inhibitory phosphorylation of Gsk3 $\beta$ (Gsk3 $\beta$ pS9), the kinase which must be inhibited by autoactivated Camk2 during the excitatory potentiation $[46,47]$.

Under resting conditions, we observed that both silencing and inhibition/tetramerization of Fbp2 had no effect on phosphorylation status of the studied proteins (Figure 5A). The induction of LTP resulted in a significant increase of the level of phosphorylation of Camk2 $\alpha$, AMPAR and Gsk3 $\beta$ indicating postsynaptic potentiation (Figure 5B-E).

However, both silencing and inhibition of $\mathrm{Fbp} 2$ almost completely blocked the phosphorylation of all the monitored proteins (Figure 5B-E) and thus, the early phase of LTP.

Since the electrophysiological studies demonstrated that silencing of Fbp2 inhibited the LTP formation (Figure 4) thus, we addressed the question whether Fbp2 defects are reflected by deficits in the expression of the late LTP markers. 


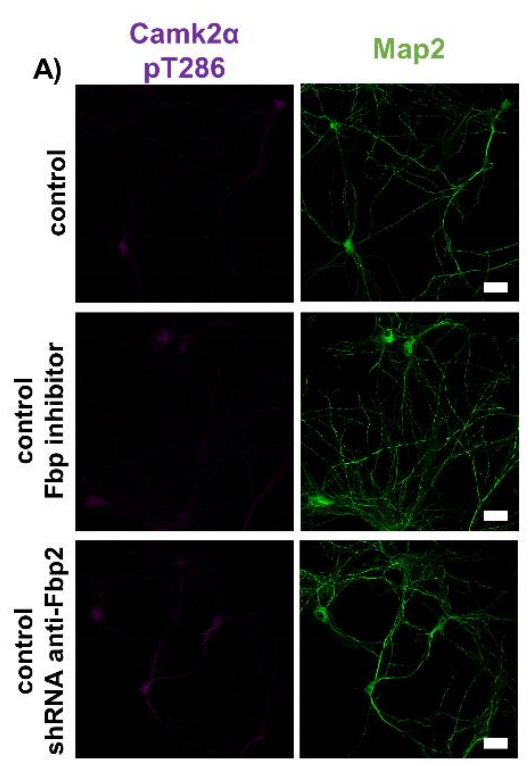

AMPAR (Gria1)

B)
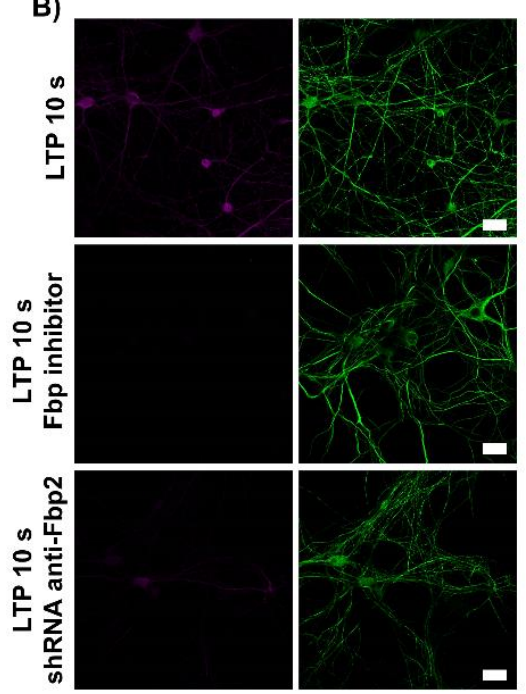

C)

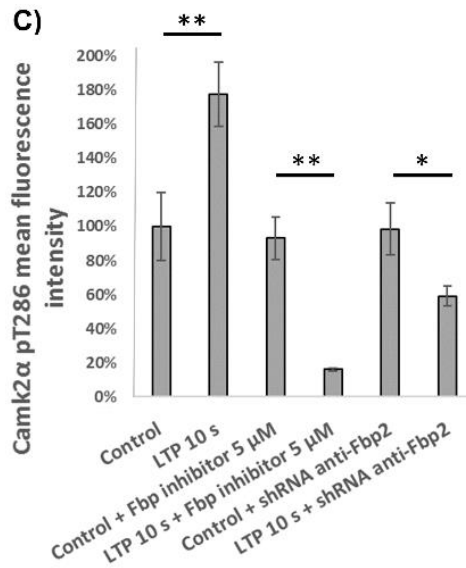

pS831

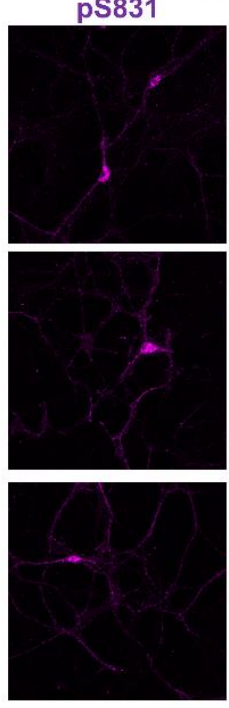

Map2
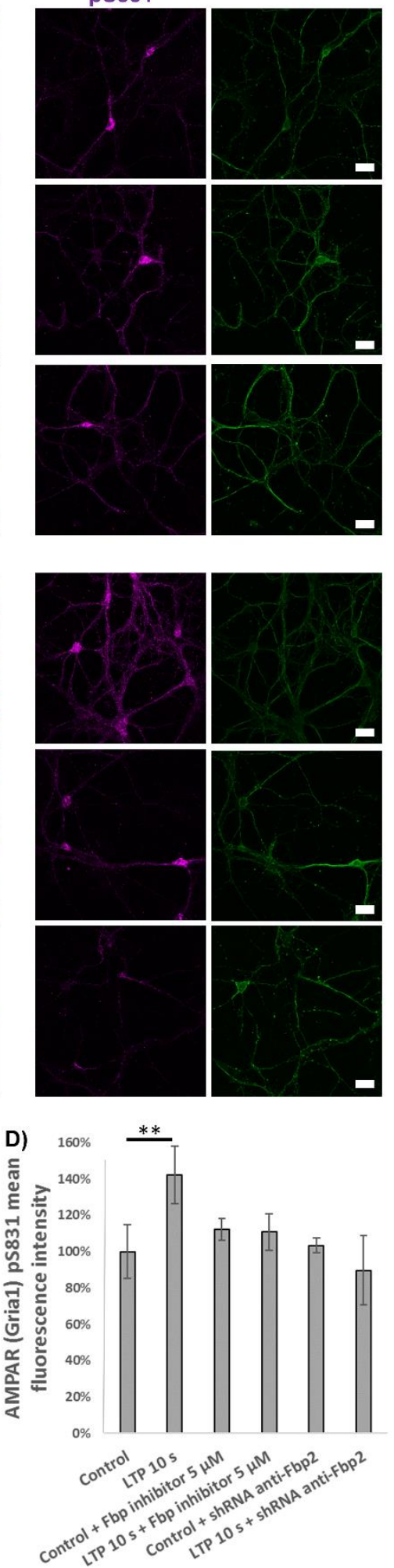
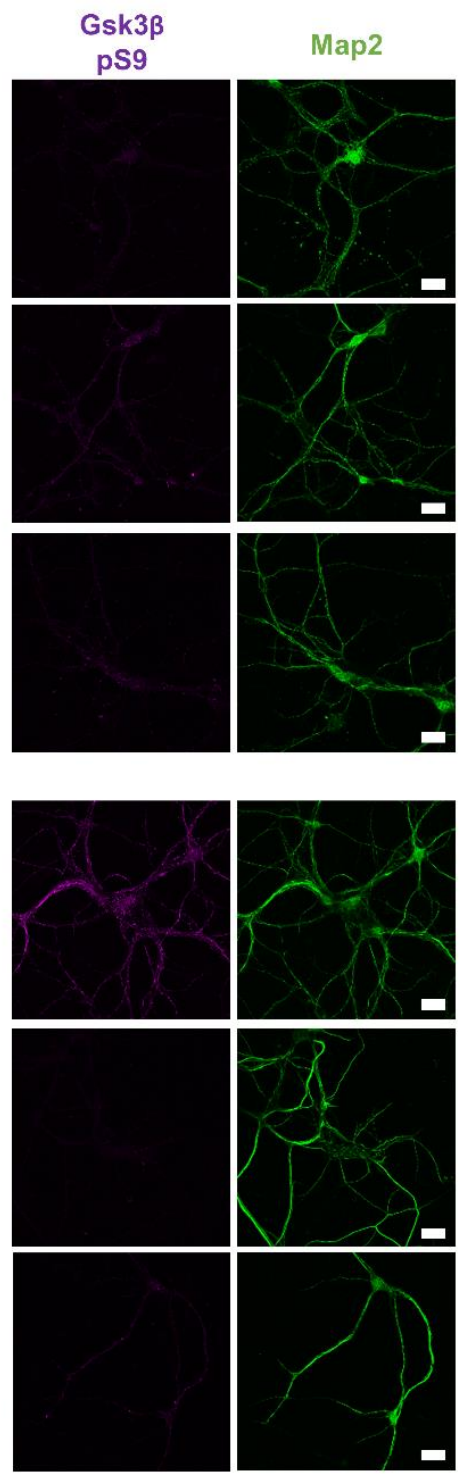

E)

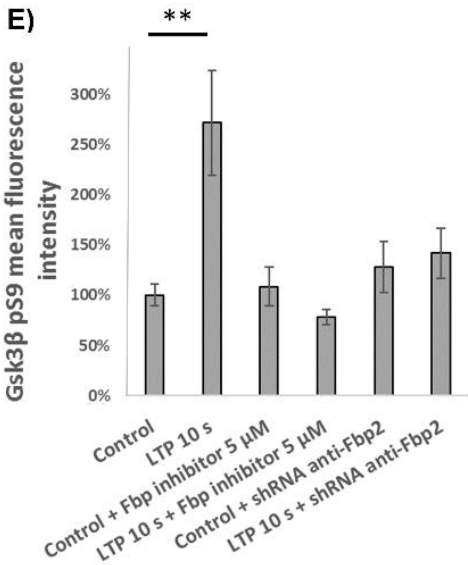

Figure 5. Silencing or inhibition/tetramerization of Fbp2 block expression of the early phase of LTP.

A) Fluorescence signals associated with phosphorylated forms of Camk2 $\alpha$, AMPAR (Gria1) and Gsk3 $\beta$ are not affected by Fbp2 silencing or inhibition/tetramerization in neurons in which LTP was not induced. B) Induction of LTP stimulates phosphorylation of Camk2 $\alpha$, AMPAR and Gsk3 $\beta$ (upper row) but manipulation with Fbp2 amount and structure strongly reduces the phosphorylation. C) Quantification of the fluorescence associated with phosphorylated forms of Camk2 $\alpha$ (pT286), AMPAR (pS831) and Gsk3 3 (pS9) as compared to the fluorescence recorded in unstimulated neurons (controls).

Bar $=20 \mu \mathrm{m}$. For each condition N=25 ROIs. The values are given as a mean and SD. * indicates $\mathrm{p}<0.05,{ }^{* *}$ indicates $\mathrm{p}<0.01$. 
We tested the expression of c-Jun and c-Fos, two transcriptional factors responsible for the formation of LTP [5], and nuclear localization of Camk4, a kinase involved in stimulation of the expression of LTP-associated transcriptional factors [48]. Our results revealed that both inhibition/tetramerization and silencing of Fbp2 almost completely abolished LTP-induced expression of the transcription factors (Figure 6A) and blocked the LTP-dependent nuclear accumulation of Camk4 (Figure 6B). This corroborates the results obtained using electrophysiological methods (Figure 4).

A)

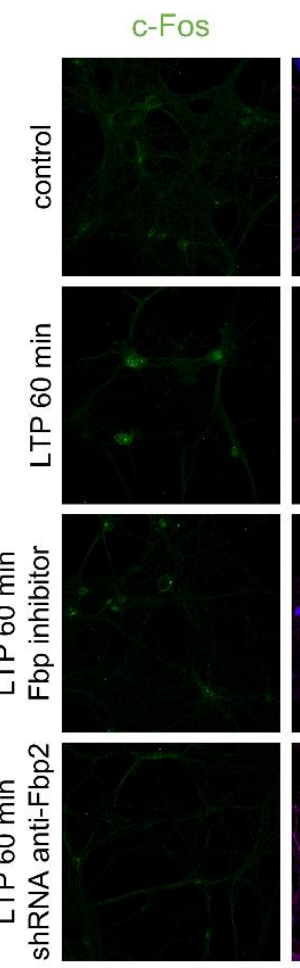

B)

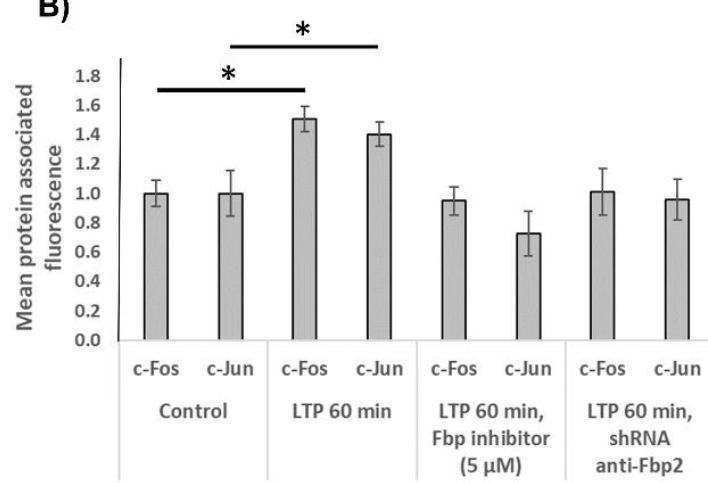

D)

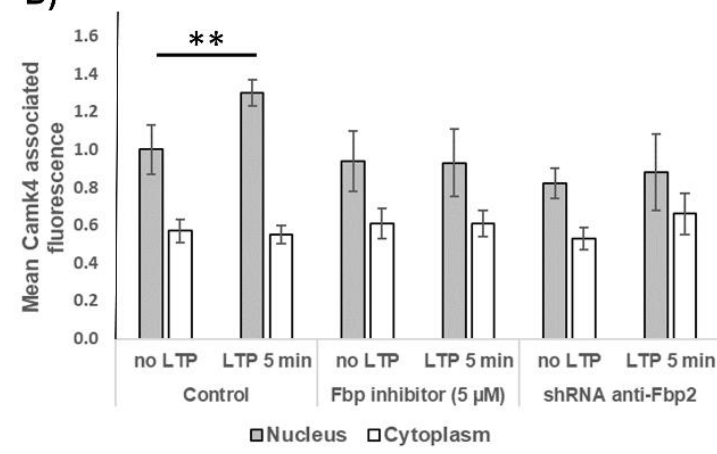

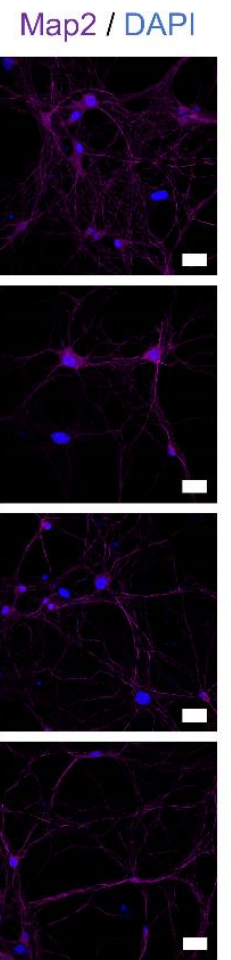

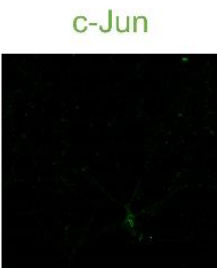

Map2 / DAP|

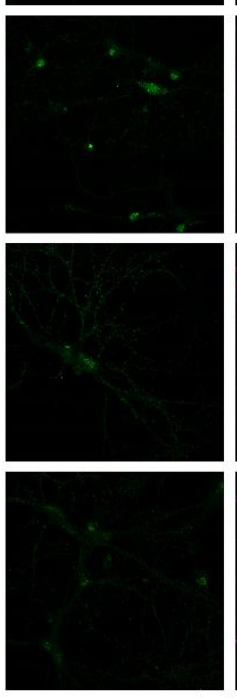

C)
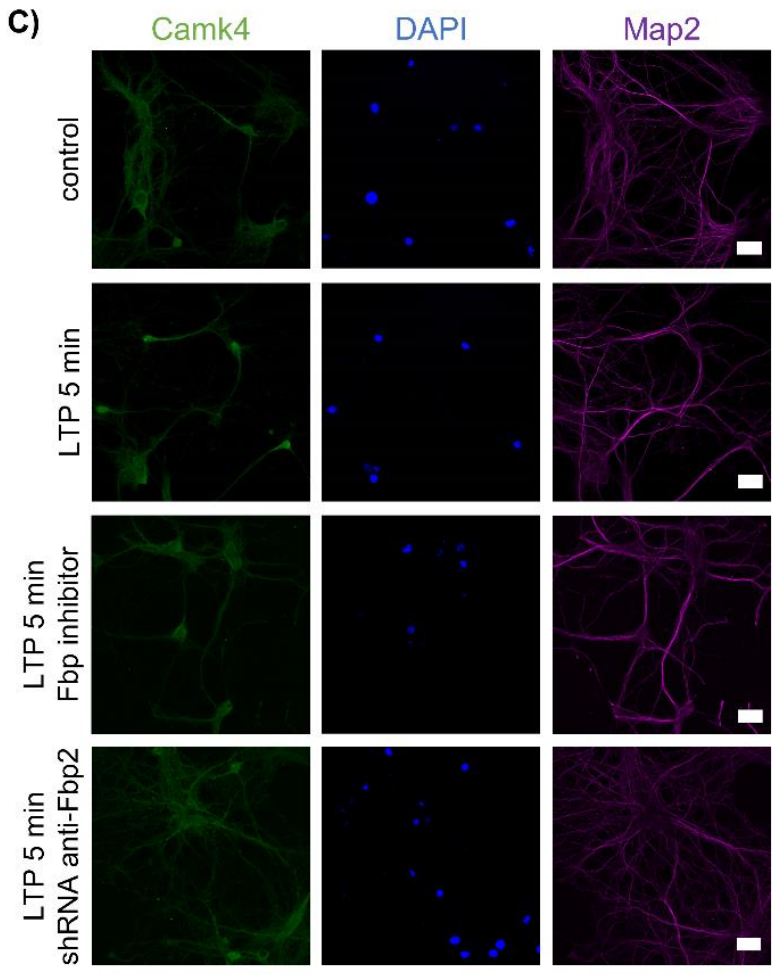
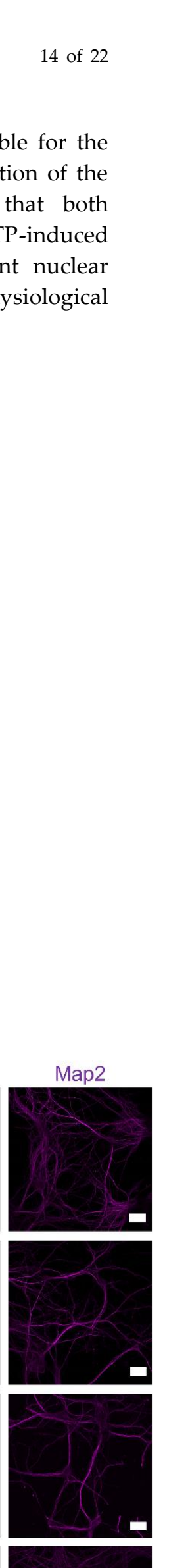
Figure 6. Expression of the late phase LTP depends on Fbp2 structure and concentration.

A) Induction of LTP increases fluorescence related to c-Fos (left panel) and c-Jun (right panel), however, inhibition of Fbp or reduced concentration of the enzyme abolish the expression of both the transcription factors. B) Quantification of mean c-Fosand c-Jun-associated fluorescence in neurons after the LTP induction as compared to the fluorescence in neurons in which LTP was not induced. For each condition $\mathrm{N}=30$ ROIs. C) Nuclear translocation of Camk4 is blocked by Fbp inhibition/tetramerization or silencing. D) Mean Camk4-associated fluorescence from neuronal nuclei after the LTP induction as compared that observed in unstimulated neurons (control). For each condition $\mathrm{N}=20$ ROIs.

Bar $=20 \mu \mathrm{m}$. The values are given as a mean and SD. ${ }^{*}$ indicates $\mathrm{p}<0.05,{ }^{* * *}$ indicates $\mathrm{p}<0.01$.

\section{Fbp2 interaction with Camk2}

Both inhibition/tetramerization and silencing of Fbp2 blocked Camk2 autoactivation during the LTP induction (Figure 5B) and led to disruption of the LTP formation (Figure 4, Figure 5B-E and Figure 6). It raised an intriguing question if $\mathrm{Fbp} 2$ is directly involved, via protein-protein interactions, in the regulation of Camk2 autophosphorylation.

The results of our co-immunoprecipitation experiment with the use of purified proteins demonstrated that Camk2 $\alpha$ and Fbp2 may associate with each other (Figure 7A). Since immunoprecipitation is a semiquantitative technique we used biolayer interferometry (BLI) and thermophoresis to describe the Fbp2-Camk2 $\alpha$ interaction.

Using the BLI we observed a very strong interaction between Fbp2 and Camk2 $\alpha$, with picomolar apparent binding constant (Figure 7B). Unexpectedly, after withdrawal of the free (unbound) Fbp2 from the assay medium we were not able to detect any dissociation of Fbp2 from Camk2 $\alpha$ for over five minutes (Figure 7B). It may confirm the significant strength of the interaction, however, it may also reflect global and long lasting structural changes within the Camk2 $\alpha$-Fbp2 complex. Additionally, we performed steady-state analysis based on the recorded data. The dissociation constant calculated from the fitted saturation binding curves equalled to $0.1 \mu \mathrm{M}$ (Figure 7C).

In the BLI method, Camk $2 \alpha$ was covalently attached to the sensor. Thus, to check if both proteins can interact with each other in solution, we used the thermophoresis (also called thermodiffusion)based technique. The results revealed that $\mathrm{Fbp} 2$ binds to Camk2 $\alpha$ with dissociation constant $\mathrm{KD}=1.2$ $\mu \mathrm{M}$ (Figure 7D).

Finally, using the Duolink technique, we verified the proteins' interaction in cultured neurons. In control conditions, we found only a low degree of colocalization of Fbp2 and Camk2 $\alpha$ (Figure 7E). However, the chemical induction of LTP resulted in a mass appearance of fluorescent signal associated with the Fbp2-Camk2 $\alpha$ complex (Figure 7E), corroborating the interaction of the proteins in living cells and its LTP-induction dependence. 

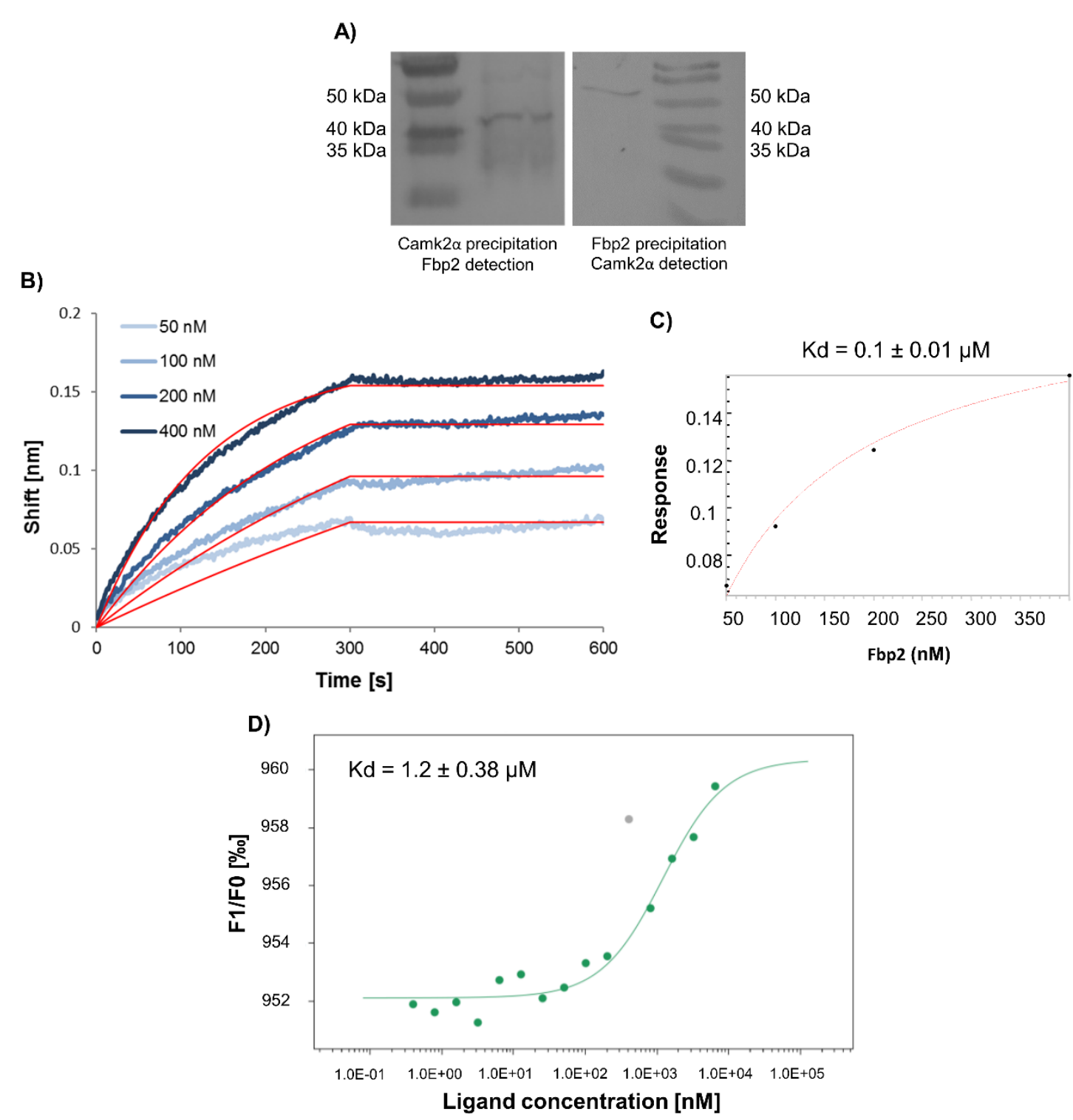

E)

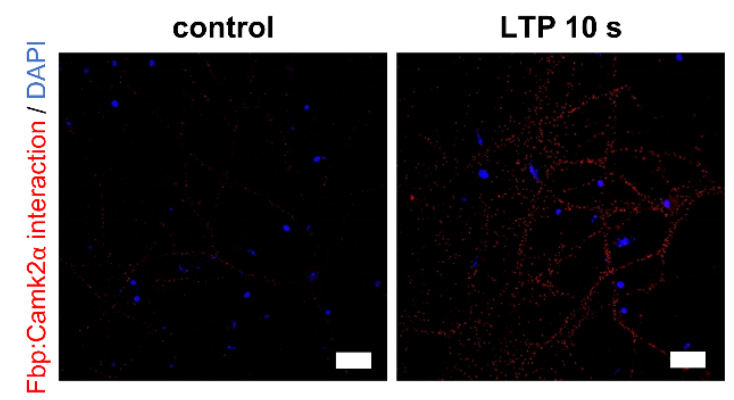

Figure 7. Fbp2 interacts with Camk2 $\alpha$.

A) Co-immunoprecipitation of Fbp2-Camk2 $\alpha$ complex; the left image shows Western blot detection of Fbp2 after precipitation of the complex with anti-Camk2 $\alpha$ antibodies, while the right - immunodetection of Camk2 $\alpha$ after the precipitation with the use of anti-Fbp IgG. B) BLI sensograms of Fbp2 binding to Camk2 $\alpha$. The red lines represent global fit to the 1:1 interaction model. C) Steady state analysis of the Fbp2-Camk2 $\alpha$ interaction. D) Fbp2 binds to Camk2 $\alpha(\mathrm{Kd}=1.2 \mu \mathrm{M})$ as quantified using microscale thermophoresis experiments. E) In situ detection of Fbp2 interaction with Camk2 $\alpha$ in 10 sec after the LTP induction. Red spots indicate the places in which the enzymes interact with each other $(B a r=20 \mu \mathrm{m})$. 


\section{Discussion}

LTP and long-term synaptic depression (LTD) are regarded as the basic mechanism of the long term memory formation. Here, we present several lines of evidence that the presence of Fbp2 is indispensable for the induction and expression of LTP.

Fbp2 is a glyconeogenic enzyme. However, neurons are not supposed to synthesize glycogen from carbohydrate precursors [49]. Moreover, expression of Fbp2 as well as its liver isoform - Fbp1, in neurons is very low. Hence, it is not likely that the enzyme may significantly contribute to any metabolic pathway. Nevertheless, Fbp2 is also involved in protection of mitochondria against calcium-induced stress in a non-enzymatic manner [7]. Since neuronal mitochondria are continuously exposed to elevated $\mathrm{Ca}^{2+}$ levels during activation of the cells, the association of $\mathrm{Fbp} 2$ to these organelles may be a mechanism protecting the organelles and whole neurons against defectivemitochondria-mediated death.

In this report, we demonstrated that about half of the Fbp2 molecules is colocalized with mitochondria in unstimulated neurons, and induction of LTP results in dynamic, $\mathrm{Ca}^{2+}$ - and mitochondrial membrane potential-dependent changes in degree of the colocalization. Generally, except the short early phase of the LTP formation (the first $\sim 8 \mathrm{sec}$ ), a majority of the enzyme is associated with mitochondria for at least $60 \mathrm{~min}$ - a period in which mitochondria are moderately depolarized with a phasic occurrence of mitoflashes, and the expression of proteins involved in the late phase of LTP is stimulated. Such a high level of the colocalization, even in unstimulated neurons, is in line with the protective role of Fbp2 in mitochondria subjected to stress stimuli.

Importantly, we also show that the presence of $\mathrm{Fbp}$ in neurons is required for initiation of the LTP formation. Fbp2 interacts with Camk2 $\alpha$ during NMDAR-dependent activity at excitatory synapses and stimulates Camk2 phosphorylation which is the first step in the LTP induction. Silencing of Fbp2 results in disruption of both the early phase of LTP (as determined by changes in phosphorylation status of Camk2, AMPAR and Gsk3) and the late phase of the memory formation (monitored by expression of such LTP-associated transcription factors as c-Fos and c-Jun and nuclear localization of Camk4).

Interestingly, the application of iFbp reproduces the effect of the enzyme silencing on the LTP formation. In a cell, Fbp2 exists in equilibrium of various oligomeric forms, predominantly dimers and tetramers [12], and binding of physiological allosteric inhibitors, such as AMP and NAD+, stabilizes the tetrameric conformation and inhibits enzymatic activity of Fbp2 [12]. Since iFbp mimics the effect of the endogenous inhibitors, it may be presumed that Fbp2 adopts the tetrameric quaternary conformation in cells treated with this compound. Thus, it could be inferred that the dimeric form of Fbp2 is the preferred form of the enzyme which interacts with Camk2 $\alpha$ and ensures the LTP formation.

The preferred binding of the dimeric Fbp2 to mitochondria and Camk2 may explain the mechanism of astrocyte-neuron lactate shuttle (ANLS) stimulation of LTP induction. ANLS denotes the process in which astrocyte-derived lactate is converted by neurons to pyruvate and used to fulfill energy requirements of neuronal cells [50,51]. Although the significance of ANLS for the whole brain energetics is under dispute [52-55] the absolute requirement for the lactate transport from astrocytes to neurons during LTP formation is not controversial $[6,55,56]$. It has been demonstrated that the astrocyte-derived lactate fuels expression and translation of memory formation-associated mRNA $[14,56]$. Moreover, it has been shown that elevation of NADH/NAD ${ }^{+}$ratio, which accompanies oxidation of lactate to pyruvate, stimulates activity of NMDAR by modifying neuronal redox state [14]. Importantly, the reduced NAD+/NADH ratio is also a factor increasing the pool of dimeric Fbp2 because $\mathrm{NAD}^{+}$stimulates the enzyme tetramerization and inactivation [12]. Thus, the uptake of astrocyte-derived lactate by neurons might be considered as one of mechanisms stimulating Fbp2 dimerization in neurons, and hence, enabling - via Camk2 activation - induction of LTP and protecting mitochondria against calcium stress.

Concluding, data presented here demonstrates that induction and formation of the NMDARdependent plasticity in hippocampal neurons and slices depends on the presence of Fbp2. 
Neighbouring astrocytes may participate in the induction of LTP in the ANLS-dependent manner, via regulation of the oligomeric state of $\mathrm{Fbp} 2$ in neurons.

Our findings open a new area of study directly linking memory formation to energy state of the brain. Further analysis of the interdependence between Fbp2 oligomerization state and interactions of the enzyme with cellular binding partners in the context of astrocyte-neuron cross-talk, is likely to reveal a precise mechanistic insight into synaptic plasticity.

The results presented here also suggest that a decreased binding of Fbp2 to mitochondria caused by reduced amount of the enzyme or presence of defective forms of Fbp2 (with impaired ability of oligomerization or reduced affinity to mitochondria) might be associated with certain mitochondrial function-related brain disorders [57].

Additionally, the proper mode of Fbp2 interaction with mitochondria depends also on Gsk3 $\beta$ inactivation. Gsk3 $\beta$ is a kinase which overactivity is attributed to Alzheimer's disease pathogenesis [58]. Thus, an attractive hypothesis is that disruption of mitochondrial function during progression of the disease [59] at least partially results from inability of Fbp2 to protect mitochondria against stress stimuli in the presence of permanently active Gsk3 $\beta$.

Finally, it is essentially unknown whether in neurons, Fbp2 expression and the enzyme posttranslational modifications change during aging, and whether such changes impact brain plasticity and lead to age-dependent memory decline.

Author Contributions: PD: investigation, formal analysis, data curation, visualization; TW: formal analysis, investigation; JJ, DK and AC: investigation; AG: conceptualization, methodology, writing - original draft, writing - review \& editing; DR: conceptualization, methodology, supervision, writing - original draft, writing review \& editing. All authors have read and agreed to the published version of the manuscript.

Funding: This research was funded by the Polish National Science Centre: UMO-2015/19/B/NZ1/00332.

Acknowledgments: We wish to thank dr Janusz Wiśniewski for assistance in protein studies.

Conflicts of Interest: The authors declare no conflict of interest.

\section{References}

1. Bliss, T.V.P.; Collingridge, G.L. A synaptic model of memory: Long-term potentiation in the hippocampus. Nature 1993, 361, 31-9.

2. Collingridge, G.L.; Isaac, J.T.R.; Wang, Y.T. Receptor trafficking and synaptic plasticity. Nat Rev Neurosci 2004, 5, 952-62.

3. Derkach, V.A.; Oh, M.C.; Guire, E.S.; Soderling, T.R. Regulatory mechanisms of AMPA receptors in synaptic plasticity. Nat Rev Neurosci 2007, 8, 101-13.

4. Cole, A.J.; Saffen, D.W.; Baraban, J.M.; Worley, P.F. Rapid increase of an immediate early gene messenger RNA in hippocampal neurons by synaptic NMDA receptor activation. Nature 1989, 340, 474-6.

5. Alberini, C.M. Transcription factors in long-term memory and synaptic plasticity. Physiol Rev 2009, 89, $121-45$.

6. Suzuki, A.; Stern, S.A.; Bozdagi, O.; Huntley, G.W.; Walker, R.H.; Magistretti, P.J;; Alberini, C.M. Astrocyte-neuron lactate transport is required for long-term memory formation. Cell 2011; 144: 810-23.

7. Gizak, A.; Pirog, M.; Rakus, D. Muscle FBPase binds to cardiomyocyte mitochondria under glycogen synthase kinase-3 inhibition or elevation of cellular Ca 2+ level. FEBS Lett 2012, 586, 13-9. 
8. Duda, P.; Janczara, J.; McCubrey, J.A.; Gizak, A.; Rakus, D. The Reverse Warburg Effect is Associated with Fbp2-Dependent Hif1 $\alpha$ Regulation in Cancer Cells Stimulated by Fibroblasts. Cells 2020, 9, E205.

9. Gizak, A.; Zarzycki, M.; Rakus, D. Nuclear targeting of FBPase in HL-1 cells is controlled by beta-1 adrenergic receptor-activated Gs protein signaling cascade. Biochim Biophys Acta-Mol Cell Res 2009, 1793, $871-7$.

10. Huangyang, P.; Li, F.; Lee, P.; Nissim, I.; Weljie, A.M.; Mancuso, A.; Li, B.; Keith, B.; Yoon, S.S.; Celeste, S.M. Fructose-1,6-Bisphosphatase 2 Inhibits Sarcoma Progression by Restraining Mitochondrial Biogenesis. Cell Metab 2020, 31, 174-88.

11. Barciszewski, J.; Wisniewski, J.; Kolodziejczyk, R.; Jaskolski, M.; Rakus, D.; Dzugaj, A. T-to-R switch of muscle fructose-1,6-bisphosphatase involves fundamental changes of secondary and quaternary structure. Acta Crystallogr Sect D Struct Biol 2016, 72, 536-50.

12. Wiśniewski, J.; Piróg, M.; Hołubowicz, R.; Dobryszycki, P.; McCubrey, J.A.; Rakus, D.; Gizak, A. Dimeric and tetrameric forms of muscle fructose-1,6-bisphosphatase play different roles in the cell. Oncotarget 2017, 8, 115420-33.

13. Rakus, D.; Tillmann, H.; Wysocki, R.; Ulaszewski, S.; Eschrich, K.; Dzugaj, A. Different sensitivities of mutants and chimeric forms of human muscle and liver fructose-1,6-bisphosphatases towards AMP. Biol Chem 2003, 384, 51-8.

14. Yang, J.; Ruchti, E.; Petit, J-M.; Jourdain, P.; Grenningloh, G.; Allaman, I.; Magistretti, P.J. Lactate promotes plasticity gene expression by potentiating NMDA signaling in neurons. Proc Natl Acad Sci U S A 2014, 111, 12228-33.

15. Löffler, T.; Al-Robaiy, S.; Bigl, M.; Eschrich, K.; Schliebs, R. Expression of fructose-1,6-bisphosphatase mRNA isoforms in normal and basal forebrain cholinergic lesioned rat brain. Int J Dev Neurosci 2001, 19, 279-85.

16. Velásquez, Z.D.; Pérez, M.; Morán, M.A.; Yanez, A.J.; Avila, J.; Slebe, J.C.; Gómez-Ramos, P. Ultrastructural localization of fructose-1,6-bisphosphatase in mouse brain. Microsc Res Tech 2011, 74, 329-36.

17. Mozrzymas, J.; Szczęsny, T.; Rakus, D. The effect of glycogen phosphorolysis on basal glutaminergic transmission. Biochem Biophys Res Commun 2011, 404, 652-5.

18. Lu, W.; Man, H.; Ju, W.; Trimble, W.S.; MacDonald, J.F.; Wang, Y.T. Activation of synaptic NMDA receptors induces membrane insertion of new AMPA receptors and LTP in cultured hippocampal neurons. Neuron 2001, 29, 243-54.

19. Chen, R-Q.; Wang, S-H.; Yao, W.; Wang, J-J.; Ji, F.; Yan, J-Z.; Ren, S-Q.; Chen, Z.; Liu, S-Y.; Lu, W. Role of glycine receptors in glycine-induced LTD in hippocampal CA1 pyramidal neurons. Neuropsychopharmacology 2011, 36, 1948-58.

20. Mamczur, P.; Borsuk, B.; Paszko, J.; Sas, Z.; Mozrzymas, J.; Wiśniewski, J.R.; Gizak, A.; Rakus, D. 
Astrocyte-neuron crosstalk regulates the expression and subcellular localization of carbohydrate metabolism enzymes. Glia 2015, 63, 328-40.

21. Gizak, A.; Dzugaj, A. FBPase is in the nuclei of cardiomyocytes. FEBS Lett 2003, 539, 51-5.

22. Brzdak, P.; Wójcicka, O.; Zareba-Koziol, M.; Minge, D.; Henneberger, C.; Wlodarczyk, J.; Mozrzymas, J.W.; Wójtowicz, T. Synaptic Potentiation at Basal and Apical Dendrites of Hippocampal Pyramidal Neurons Involves Activation of a Distinct Set of Extracellular and Intracellular Molecular Cues. Cereb Cortex 2019, 29, 283-304.

23. Brzdąk, P.; Włodarczyk, J.; Mozrzymas, J.W.; Wójtowicz, T. Matrix Metalloprotease 3 Activity Supports Hippocampal EPSP-to-Spike Plasticity Following Patterned Neuronal Activity via the Regulation of NMDAR Function and Calcium Flux. Mol Neurobiol 2017, 54, 804-16.

24. Roszkowska, M.; Skupien, A.; Wójtowicz, T.; Konopka, A.; Gorlewicz, A.; Kisiel, M.; Bekisz, M.; Ruszczycki, B.; Dolezyczek, H.; Rejmak, E.; Knapska, E.; Mozrzymas, J.W.; Wlodarczyk, J.; Wilczynski, G.M.; Dzwonek, J. CD44: a novel synaptic cell adhesion molecule regulating structural and functional plasticity of dendritic spines. Mol Biol Cell 2016, 27, 4055-66.

25. Schneider, C.A.; Rasband, W.S.; Eliceiri, K.W. NIH Image to ImageJ: 25 years of image analysis. Nat Methods 2012, 9, 671-5.

26. Egashira, Y.; Mori, Y.; Yanagawa, Y.; Takamori, S. Development of lentiviral vectors for efficient glutamatergic-selective gene expression in cultured hippocampal neurons. Sci Rep 2018, 8, 15156.

27. Bolte, S.; Cordelières, F.P. A guided tour into subcellular colocalization analysis in light microscopy. $J$ Microsc 2006, 224, 213-32.

28. Pirog, M.; Gizak, A.; Rakus, D. Changes in quaternary structure of muscle fructose-1,6-bisphosphatase regulate affinity of the enzyme to mitochondria. Int J Biochem Cell Biol 2014, 48, 55-9.

29. Luscher, C.; Malenka, R.C. NMDA Receptor-Dependent Long-Term Potentiation and Long-Term Depression (LTP/LTD). Cold Spring Harb Perspect Biol 2012, 4, a005710-a005710.

30. Hongpaisan, J.; Winters, C.A.; Andrews, S.B. Strong calcium entry activates mitochondrial superoxide generation, upregulating kinase signaling in hippocampal neurons. J Neurosci 2004, 24, 10878-87.

31. Divakaruni, S.S.; Van Dyke, A.M.; Chandra, R.; LeGates, T.A.; Contreras, M.; Dharmasri, P.A.; Higgs, H.N.; Lobo, M.K.; Thompson, S.M.; Blanpied, T.A. Long-Term Potentiation Requires a Rapid Burst of Dendritic Mitochondrial Fission during Induction. Neuron 2018, 100, 860-875.e7.

32. Harvey, J.; Collingridge, G.L. Thapsigargin blocks the induction of long-term potentiation in rat hippocampal slices. Neurosci Lett 1992, 139, 197-200.

33. De J García-Rivas, G.; Carvajal, K.; Correa, F.; Zazueta, C. Ru 360, a specific mitochondrial calcium uptake inhibitor, improves cardiac post-ischaemic functional recovery in rats in vivo. Br J Pharmacol 2006, $149,829-37$. 
34. Sehgal, P.; Szalai, P.; Olesen, C.; Praetorius, H.A.; Nissen, P.; Christensen, S.B.; Engedal, N.; Møller, J. V. Inhibition of the sarco/endoplasmic reticulum (ER) Ca2-ATPase by thapsigargin analogs induces cell death via ER Ca2 depletion and the unfolded protein response. J Biol Chem 2017, 292, 19656-73.

35. Malenka, R.C. Synaptic plasticity in the hippocampus: LTP and LTD. Cell 1994, 78, 535-8.

36. Matias, C.; Dionísio, J.C.; Quinta-Ferreira, M.E. Thapsigargin blocks STP and LTP related calcium enhancements in hippocampal CAI area. Neuroreport 2002, 13, 2577-80.

37. Kim, H.Y.; Lee, K.Y.; Lu, Y.; Wang, J.; Cui, L.; Kim, S.J.; Chung, J.M.; Chung, K. Mitochondrial Ca2+ uptake is essential for synaptic plasticity in pain. J Neurosci 2011, 31, 12982-91.

38. Barish, M.E.; Ichikawa, M.; Tominaga, T.; Matsumoto, G.; Iijima, T. Enhanced fast synaptic transmission and a delayed depolarization induced by transient potassium current blockade in rat hippocampal slice as studied by optical recording. J Neurosci 1996, 16, 5672-87.

39. Fu, Z-X.; Tan, X.; Fang, H.; Lau, P-M.; Wang, X.; Cheng, H.; Bi, G-Q. Dendritic mitoflash as a putative signal for stabilizing long-term synaptic plasticity. Nat Commun 2017, 8, 31.

40. Peng, T.I.; Greenamyre, J.T. Privileged access to mitochondria of calcium influx through N-methyl-Daspartate receptors. Mol Pharmacol 1998, 53, 974-80.

41. Gizak, A.; Duda, P.; Wisniewski, J.; Rakus, D. Fructose-1,6-bisphosphatase: From a glucose metabolism enzyme to multifaceted regulator of a cell fate. Adv Biol Regul 2019, 72, 41-50.

42. Lai, C.; Gum, R.J.; Daly, M.; Fry, E.H.; Hutchins, C.; Abad-Zapatero, C.; von Geldern, T.W. Benzoxazole benzenesulfonamides as allosteric inhibitors of fructose-1,6-bisphosphatase. Bioorg Med Chem Lett 2006, $16,1807-10$.

43. von Geldern, T.W.; Lai, C.; Gum, R.J.; Daly, M.; Sun, C.; Fry, E.H.; Abad-Zapatero, C. Benzoxazole benzenesulfonamides are novel allosteric inhibitors of fructose-1,6-bisphosphatase with a distinct binding mode. Bioorg Med Chem Lett 2006, 16, 1811-5.

44. Chang, J.Y.; Parra-Bueno, P.; Laviv, T.; Szatmari, E.M.; Lee, S.J.R.; Yasuda, R. CaMKII Autophosphorylation Is Necessary for Optimal Integration of Ca2+ Signals during LTP Induction, but Not Maintenance. Neuron 2017, 94, 800-808.e4.

45. Diering, G.H.; Heo, S.; Hussain, N.K.; Liu, B.; Huganir, R.L. Extensive phosphorylation of AMPA receptors in neurons. Proc Natl Acad Sci U S A 2016, 113, E4920-7.

46. Zhu, L.Q.; Wang, S.H.; Liu, D.; Yin, Y.Y.; Tian, Q.; Wang, X.C.; Wang, Q.; Chen, J.G.; Wang, J.Z. Activation of glycogen synthase kinase-3 inhibits long-term potentiation with synapse-associated impairments. J Neurosci 2007, 27, 12211-20.

47. Song, B.; Lai, B.; Zheng, Z.; Zhang, Y.; Luo, J.; Wang, C.; Chen, Y.; Woodgett, J.R.; Li, M. Inhibitory Phosphorylation of GSK-3 by CaMKII Couples Depolarization to Neuronal Survival. J Biol Chem 2010, $285,41122-34$. 
48. Kasahara, J.; Fukunaga, K.; Miyamoto, E. Activation of Calcium/Calmodulin-dependent Protein Kinase IV in Long Term Potentiation in the Rat Hippocampal CA1 Region. J Biol Chem 2001, 276, 24044-50.

49. Brown, A.M. Brain glycogen re-awakened. J Neurochem 2004, 89, 537-52.

50. Pellerin, L.; Magistretti, P.J. Glutamate uptake into astrocytes stimulates aerobic glycolysis: a mechanism coupling neuronal activity to glucose utilization. Proc Natl Acad Sci U S A 1994, 91, 10625-9.

51. Pellerin, L.; Magistretti, P.J. Sweet sixteen for ANLS. J Cereb Blood Flow Metab 2012, 32, 1152-66.

52. Lundgaard, I.; Li, B.; Xie, L.; Kang, H.; Sanggaard, S.; Haswell, J.D.R.; Sun, W.; Goldman, S.; Blekot, S.; Nielsen, M.; Takano, T.; Deane, R.; Nedergaard, M. Direct neuronal glucose uptake heralds activitydependent increases in cerebral metabolism. Nat Commun 2015, 6, 6807.

53. Dienel, G.A. Lack of appropriate stoichiometry: Strong evidence against an energetically important astrocyte-neuron lactate shuttle in brain. J Neurosci Res 2017, 95, 2103-25.

54. Mächler, P.; Wyss, M.T.; Elsayed, M.; Stobart, J.; Gutierrez, R.; Von Faber-Castell, A.; Kaelin, V.; Zuend, M.; San Martín, A.; Romero-Gómez, I.; Baeza-Lehnert, F.; Lengacher, S.; Schneider, B.L.; Aebischer, P.; Magistretti, P.J.; Barros, L.F.; Weber, B. In Vivo Evidence for a Lactate Gradient from Astrocytes to Neurons. Cell Metab 2016, 23, 94-102.

55. Drulis-Fajdasz, D.; Gizak, A.; Wójtowicz, T.; Wiśniewski, J.R.; Rakus, D. Aging-associated changes in hippocampal glycogen metabolism in mice. Evidence for and against astrocyte-to-neuron lactate shuttle. Glia 2018, 66, 1481-95.

56. Descalzi, G.; Gao, V.; Steinman, M.Q.; Suzuki, A.; Alberini, C.M. Lactate from astrocytes fuels learninginduced mRNA translation in excitatory and inhibitory neurons. Commun Biol 2019, 2, 247.

57. Dagda, R.K. Role of Mitochondrial Dysfunction in Degenerative Brain Diseases, an Overview. Brain Sci 2018, 8, 178 .

58. Lauretti, E.; Dincer, O.; Praticò, D. Glycogen synthase kinase-3 signaling in Alzheimer's disease. Biochim Biophys Acta Mol Cell Res 2020, 1867, 118664.

59. Moreira, P.I.; Carvalho, C.; Zhu, X.; Smith, M.A.; Perry, G. Mitochondrial dysfunction is a trigger of Alzheimer's disease pathophysiology. Biochim Biophys Acta 2010, 1802, 2-10. 